\title{
Pressure-induced ferromagnetism in the topological semimetal $\mathrm{EuCd} \mathbf{A s}_{2}$
}

\author{
Elena Gati $\odot,{ }^{1,2,3,}$, Sergey L. Bud'ko, ${ }^{1,2}$ Lin-Lin Wang, ${ }^{1}$ Adrian Valadkhani, ${ }^{4}$ Ritu Gupta ${ }^{5}$ Brinda Kuthanazhi, ${ }^{1,2}$ Li Xiang, ${ }^{1,2}$ \\ John M. Wilde $\odot,{ }^{1,2}$ Aashish Sapkota, ${ }^{1,2}$ Zurab Guguchia, ${ }^{5}$ Rustem Khasanov, ${ }^{5}$ Roser Valentí ${ }^{4}{ }^{4}$ and Paul C. Canfield ${ }^{1,2, \dagger}$ \\ ${ }^{1}$ Ames Laboratory, U.S. Department of Energy, Iowa State University, Ames, Iowa 50011, USA \\ ${ }^{2}$ Department of Physics and Astronomy, Iowa State University, Ames, Iowa 50011, USA \\ ${ }^{3}$ Max Planck Institute for Chemical Physics of Solids, 01187 Dresden, Germany \\ ${ }^{4}$ Institute for Theoretical Physics, Goethe-Universität Frankfurt, Max-von-Laue-Strasse 1, 60438 Frankfurt am Main, Germany \\ ${ }^{5}$ Laboratory for Muon Spin Spectroscopy, Paul Scherrer Institute, 5232 Villigen PSI, Switzerland
}

(Received 4 August 2021; revised 27 September 2021; accepted 4 October 2021; published 13 October 2021)

\begin{abstract}
The antiferromagnet and semimetal $\mathrm{EuCd}_{2} \mathrm{As}_{2}$ has recently attracted a lot of attention due to a wealth of topological phases arising from the interplay of topology and magnetism. In particular, the presence of a single pair of Weyl points is predicted for a ferromagnetic configuration of Eu spins along the $c$ axis in $\operatorname{EuCd}_{2} \mathrm{As}_{2}$. In the search for such phases, we investigate here the effects of hydrostatic pressure in $\mathrm{EuCd}_{2} \mathrm{As}_{2}$. For that, we present specific heat, transport, and $\mu \mathrm{SR}$ measurements under hydrostatic pressure up to $\sim 2.5 \mathrm{GPa}$, combined with ab initio density functional theory (DFT) calculations. Experimentally, we establish that the ground state of $\mathrm{EuCd}_{2} \mathrm{As}_{2}$ changes from in-plane antiferromagnetic $\left(\mathrm{AFM}_{a b}\right)$ to ferromagnetic at a critical pressure of $\approx 2 \mathrm{GPa}$, which is likely characterized by the moments dominantly lying within the $a b$ plane $\left(\mathrm{FM}_{a b}\right)$. The $\mathrm{AFM}_{a b}-\mathrm{FM}_{a b}$ transition at such a relatively low pressure is supported by our DFT calculations. Furthermore, our theoretical results indicate that $\mathrm{EuCd}_{2} \mathrm{As}_{2}$ moves closer to the sought-for $\mathrm{FM}_{c}$ state (moments $\| c$ ) with increasing pressure further. We predict that a pressure of $\approx 23 \mathrm{GPa}$ will stabilize the $\mathrm{FM}_{c}$ state if Eu remains in a 2+ valence state. Thus, our work establishes hydrostatic pressure as a key tuning parameter that (i) allows for a continuous tuning between magnetic ground states in a single sample of $\mathrm{EuCd}_{2} \mathrm{As}_{2}$ and (ii) enables the exploration of the interplay between magnetism and topology and thereby motivates a series of future experiments on this magnetic Weyl semimetal.
\end{abstract}

DOI: 10.1103/PhysRevB.104.155124

\section{INTRODUCTION}

Topological materials have been at the center of many research activities in recent years due to the presence of a plethora of exotic phenomena of relevance not only from a fundamental perspective [1] but for applications [2-6] as well. In this context, Weyl semimetals $[3,4,7]$ have emerged as particularly multifaceted realizations of topological materials displaying anomalous transport phenomena, including the anomalous Hall effect [8,9], negative longitudinal magnetoresistance [10], and nonlocal transport [11].

A Weyl semimetal can be created from a Dirac semimetal by breaking either inversion symmetry [12] or time reversal symmetry $[3,4,7,13]$. Thus, Weyl nodes can only be found either in noncentrosymmetric crystals or in magnetic materials, providing a seemingly straightforward path to identify new

\footnotetext{
*elena.gati@cpfs.mpg.de

†canfield@ameslab.gov
}

Published by the American Physical Society under the terms of the Creative Commons Attribution 4.0 International license. Further distribution of this work must maintain attribution to the author(s) and the published article's title, journal citation, and DOI. Open access publication funded by the Max Planck Society.
Weyl semimetals. However, in real materials, the observation of the intriguing features of Weyl physics is often complicated $[14,15]$ by (i) additional nontopological Fermi surface pockets and (ii) the occurrence of multiple Weyl nodes. To overcome the latter issue, research is recently focusing on magnetic systems, since the breaking of time-reversal symmetry allows, in principle, the realization of a single pair of Weyl nodes [7], which, in the presence of inversion symmetry, have to be located at the same energy. In contrast, when the crystal structure breaks inversion symmetry, the minimal number of Weyl nodes is 4 , although it often is found to be higher, such as 24 in the TaAs structural family of compounds $[3,16,17]$.

Whereas several magnetic Weyl semimetals were proposed and studied experimentally [18-23], there is a continuing interest in identifying new candidate materials, which display unambiguous signatures of Weyl physics, free from the abovementioned complexities. Surveys of space groups [24] and band structure calculations [25] have conditionally identified $\mathrm{EuCd}_{2} \mathrm{As}_{2}$ (centrosymmetric space group $P \overline{3} m 1$ [26]) as a candidate for magnetic Weyl semimetal if it orders ferromagnetically (FM) with the moments parallel to the $c$ axis. In such a FM state, $\mathrm{EuCd}_{2} \mathrm{As}_{2}$ would show the minimal number of Weyl nodes in close proximity to the Fermi level without any further manipulation, such as the application of magnetic field. 
However, experimental studies indicate that the Eu spins in $\mathrm{EuCd}_{2} \mathrm{As}_{2}$ order antiferromagnetically (AFM) at ambient pressure below $T_{\mathrm{N}} \simeq 9 \mathrm{~K}[27,28]$. Although controversially discussed, most papers refer to the AFM structure as the Atype, i.e., FM layers which stack in an AFM fashion along the $c$ axis, with the moment direction confined in the $a b$ plane [28,29]. Albeit this order breaks the in-plane threefold symmetry and thus hinders the formation of Weyl nodes, there are several experimental observations that indicate that Weyl physics can manifest itself in $\mathrm{EuCd}_{2} \mathrm{As}_{2}$ due to the proximity to ferromagnetism. Among those are, e.g., the report of a single pair of Weyl nodes in $\mathrm{EuCd}_{2} \mathrm{As}_{2}$ in the $c$ polarized state that is stabilized by very moderate magnetic fields [29], as well as the claim of Weyl nodes in the paramagnetic state whose presence was attributed to quasistatic and quasi-longrange ferromagnetic fluctuations [30]. Overall, these results underline that $\mathrm{EuCd}_{2} \mathrm{As}_{2}$ is a promising host for investigations of the interplay of topology and magnetism [31]. This notion has been further supported by transport measurements at temperatures around the antiferromagnetic transition [32].

Actually, it was recently shown that small differences in the synthesis procedure of this compound can result in samples of $\mathrm{EuCd}_{2} \mathrm{As}_{2}$ with $\mathrm{FM}$ order and moments lying in the $a b$ plane [33-36]. Whereas this explicitly demonstrates that a FM state in $\mathrm{EuCd}_{2} \mathrm{As}_{2}$ is very close (in some compositional space) to the AFM one, and suggests a high degree of tunability of the magnetic properties of $\mathrm{EuCd}_{2} \mathrm{As}_{2}$, this also can cause severe complications in comparing published results on different crystals grown via different routes.

Here, we report the effect of hydrostatic pressure $p$ up to $2.5 \mathrm{GPa}$ on the magnetic properties of AFM EuCd $\mathrm{As}_{2}$ by presenting specific heat, transport, and $\mu \mathrm{SR}$ measurements, combined with DFT calculations of total energies of different magnetic configurations up to $25 \mathrm{GPa}$. Our experiments unambiguously identify a transition to an order with a pronounced FM component, which we refer to as FM order hereafter, at $p_{c} \approx 2 \mathrm{GPa}$. This FM order is likely characterized by an in-plane orientation of the magnetic moments, as supported by our DFT calculations. Further, our calculations predict a change of the moment orientation at $\approx 23 \mathrm{GPa}$ to an out-of-plane configuration, which is the required ground state for realizing a single pair of Weyl nodes in this material. Our results therefore clearly identify pressure as an experimental tuning parameter that allows for studies of the correlation of the topological properties of $\mathrm{EuCd}_{2} \mathrm{As}_{2}$ with a change of its magnetic ground state in a single sample.

The paper is organized as follows. Section II summarizes the experimental settings and theoretical methods used in this work. In Sec. III, we discuss our results, starting with a discussion of experimental phase diagram (Sec. III A), followed by a presentation of our $\mu \mathrm{SR}$ data (Sec. III B) and anisotropic magnetoresistance data (Sec. III C), and conclude with a discussion of our results from DFT calculations (Sec. IIID). Finally, in Sec. IV, we present our conclusions and an outlook.

\section{METHODS}

Single crystals of $\mathrm{EuCd}_{2} \mathrm{As}_{2}$ were grown out of $\mathrm{Sn}$ flux with the following procedure: The elements with an initial stoichiometry of Eu:Cd:As:Sn = 1:2:2:10 were put into a fritted alumina crucible [37] (sold by LSP Ceramics as a Canfield Crucible Set [38]) and sealed in a fused silica tube under a partial pressure of argon. The thus prepared ampoule was heated up to $900^{\circ} \mathrm{C}$ over 24 hours and held there for 20 hours. This was followed by a slow cooling to $550{ }^{\circ} \mathrm{C}$ over 200 hours and decanting of the excess flux using a centrifuge [39]. The crystals were characterized by the means of powder x-ray diffraction as well as magnetic measurements (see Ref. [33] for the results). The latter measurements confirmed that these crystals undergo an AFM transition [33] at $T_{\mathrm{N}} \sim 9.5 \mathrm{~K}$ and are consistent with the A-type AFM structure.

Specific heat under pressure was measured on a single crystal using the AC calorimetry technique, as described in detail in Ref. [40]. As outlined in this reference, changes of the absolute values of the specific heat with pressure can be determined reliably up to $5-10 \%$ with this setup. Resistance under pressure was measured in a four-point configuration. Contacts were made using Epo-tek H20E silver epoxy. Unfortunately, despite using different contact materials and routes of surface preparation, it was not possible to get two-point resistances smaller than several tens of Ohms. Given that we are mostly interested in tracking anomalies in the temperature and field dependence of $R(T)$, which we carefully measure by reducing the measurement current, we refrain from correcting the presented transport data for geometrical factors. For specific heat as well as transport measurements, the cryogenic environment was provided by a Quantum Design Physical Property Measurement System. Pressure was generated in a piston-cylinder double-wall pressure cell with the outer cylinder made out of $\mathrm{CuBe}$ and the inner cylinder out of $\mathrm{Ni}-\mathrm{Cr}-\mathrm{Al}$ alloy (see Ref. [41] for a very similar design). A mixture of 4:6 light mineral oil:n-pentane was used as a pressuretransmitting medium. This medium solidifies at $p \approx 3-4 \mathrm{GPa}$ at room temperature [42], thus ensuring hydrostatic pressure application over the available pressure range. Pressure at low temperatures was determined from the shift of the superconducting transition temperature of elemental lead $(\mathrm{Pb})$ [43]. For measurements in finite magnetic field, a small misalignment (up to $5^{\circ}$ ) of the sample with respect to the denoted field orientation cannot be excluded. At each temperature, field was changed from $-9 \mathrm{~T}$ to $9 \mathrm{~T}$ and in the following, the even component was calculated to extract the magnetoresistance data.

$\mu \mathrm{SR}$ measurements under pressure were performed in a ${ }^{3} \mathrm{He}$ cryostat at the $\mu \mathrm{E} 1$ beamline at the Paul-ScherrerInstitute in Villigen, Switzerland by using the GPD spectrometer. Typically, 5-10 $\times 10^{6}$ positron events were counted for each data point. A large number of single crystals of total mass of $\approx 2 \mathrm{~g}$ were placed inside a pressure cell with arbitrary orientations. Both the inner and the outer cylinder of the pressure cell are made out of MP35N alloy [44]. Daphne 7373 oil was used as a pressure-transmitting medium, which solidifies at room temperature close to $2.5 \mathrm{GPa}$ [42]. The pressure at low temperatures was determined from the shift of the superconducting transition of elemental indium [45], which was also placed in the pressure cell and measured in an independent ac susceptibility experiment.

Total energies for $\mathrm{EuCd}_{2} \mathrm{As}_{2}$ with various Eu spin configurations were calculated in DFT [46,47] including spin-orbit coupling (SOC) with the Perdew-Burke-Ernzerhof (PBE) 
exchange-correlation functional [48]. We employed a planewave basis set and projector augmented wave (PAW) [49,50] method as implemented in VASP [51-53]. To account for the half-filled strongly localized Eu $4 f$ orbitals, a Hubbard-like $U$ parameter of $4.4 \mathrm{eV}$ was used which places the $f$ states in the region of $-0.8 \mathrm{eV}$ to $-1.4 \mathrm{eV}$, as observed experimentally in photoemission experiments [30]. The analysis of the magnetism as a function of pressure was done by performing equation of state (EOS) calculations of both A-type antiferromagnetic and in-plane ferromagnetic $\mathrm{EuCd}_{2} \mathrm{As}_{2}$ with the primitive hexagonal unit cell doubled along the $c$ axis (note that the energies of different moment directions in the plane were so close that differences could not be resolved within DFT). The corresponding reciprocal lattice was sampled on a $\Gamma$-centered Monkhorst-Pack [54] $(11 \times 11 \times 3) k$-point mesh with a Gaussian smearing of $0.05 \mathrm{eV}$. We used a kinetic energy cutoff of $318 \mathrm{eV}$ to relax the shape of the unit cell and atomic positions at selected volumes until the absolute force on each atom is below $0.01 \mathrm{eV} / \AA$. Specifically for obtaining the crystalline magnetoanisotropy calculations, we increased the $k$-point mesh to $(22 \times 22 \times 6)$, the kinetic energy cutoff was set to $500 \mathrm{eV}$, and the absolute force threshold was set to $0.001 \mathrm{eV} / \AA ̊ \AA$ per atom. In Appendix A 5 we present a discussion on the effects that the choice of initial settings in the DFT calculations have on the final relaxed structures.

\section{RESULTS AND DISCUSSION}

\section{A. Zero-field temperature-pressure phase diagram}

First, we discuss our determination of the temperaturepressure phase diagram of $\mathrm{EuCd}_{2} \mathrm{As}_{2}$ in zero field from specific heat $C / T$ and resistance $R$ measurements. Figure 1 shows $C / T$ data as a function of temperature in three pressure ranges up to $2.43 \mathrm{GPa}$. Close to ambient pressure, as represented by the $0.15 \mathrm{GPa}$ data in Fig. 1(a), we observe a sharp transition at $\sim 9.4 \mathrm{~K}$ which signals the onset of antiferromagnetic order, consistent with literature [33]. Upon increasing pressure up to $1.30 \mathrm{GPa}$ [Fig. 1(a)], this feature shifts to higher temperatures, whereas almost no change can be found in the shape of the feature, i.e., in the sharpness and the maximum value of $C / T$ at the transition. Further increasing pressure beyond $1.30 \mathrm{GPa}$ [Fig. 1(b)] results in a clear decrease of the transition temperature up to $\sim 1.94 \mathrm{GPa}$. Concurrently, the specific heat feature starts to broaden slightly and the maximum value decreases, implying that the associated entropy release with the magnetic ordering is distributed over a wider temperature range. At even higher pressures, up to the maximum pressure of our experiment of $2.43 \mathrm{GPa}$ [Fig. 1(c)], the transition temperature increases again and the increase takes place at a faster rate compared to lower pressures. The specific heat feature is significantly broadened, indicating that fluctuations might play a role in a wider temperature range above the transition temperature. The observations described above, such as the sharp feature in the temperature-pressure phase diagram at $\approx 2 \mathrm{GPa}$ (see also Fig. 3 below), suggest a change of the magnetic structure of $\mathrm{EuCd}_{2} \mathrm{As}_{2}$ with hydrostatic pressure.

To complement our thermodynamic analysis of the phase diagram, we also performed measurements of the resistance

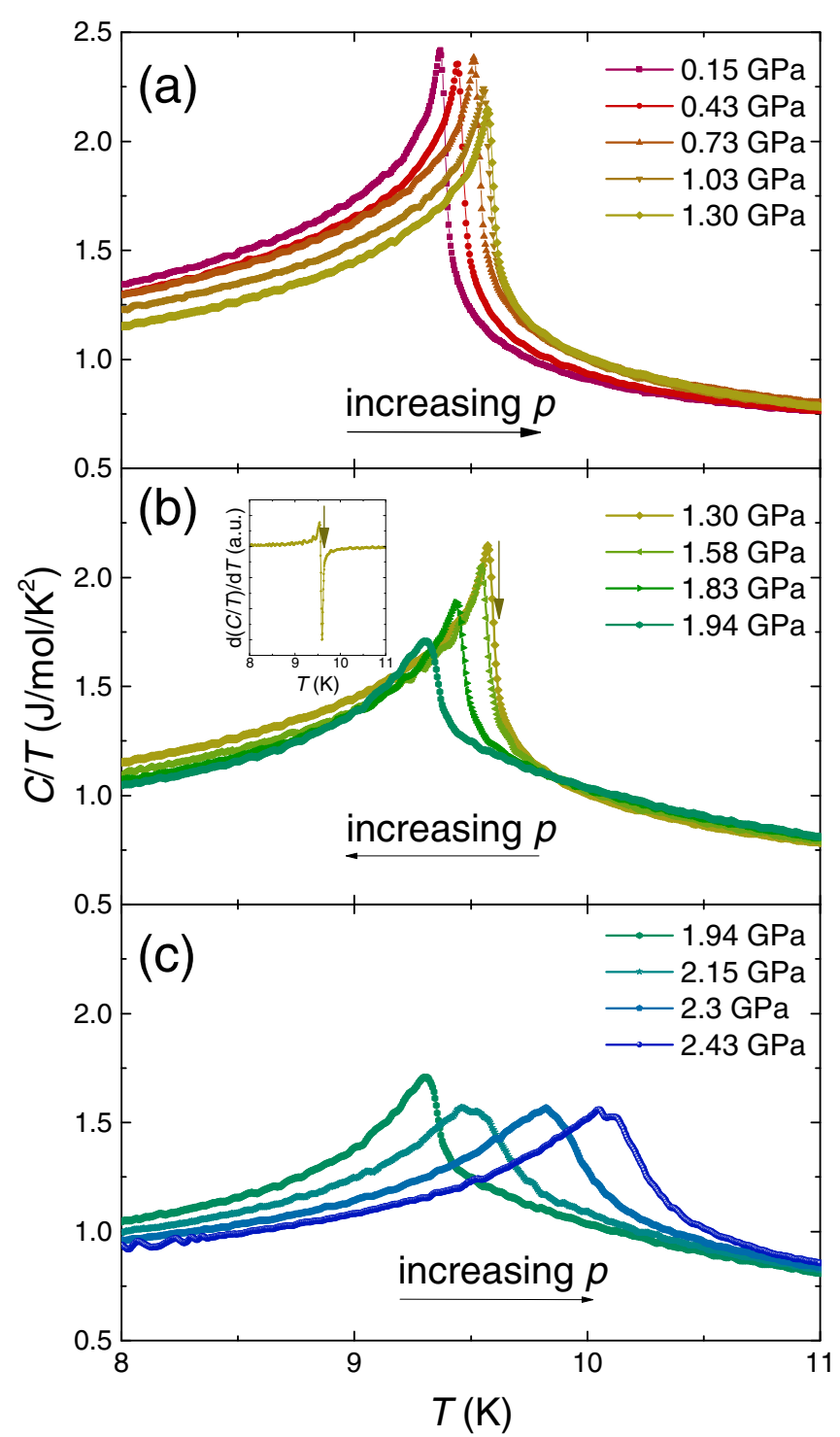

FIG. 1. Selected data sets of specific heat divided by temperature, $C / T$, vs $T(8 \mathrm{~K} \leqslant T \leqslant 11 \mathrm{~K})$ of $\mathrm{EuCd}_{2} \mathrm{As}_{2}$ under pressure $p$ for $0.13 \mathrm{GPa} \leqslant p \leqslant 1.30 \mathrm{GPa}(\mathrm{a})$, for $1.30 \mathrm{GPa} \leqslant p \leqslant 1.94 \mathrm{GPa}$ (b), and $1.94 \mathrm{GPa} \leqslant p \leqslant 2.43 \mathrm{GPa}$ (c). The inset in (b) shows an example data set of $d(C / T) / d T$, the minimum of which is used to determine the transition temperature (see arrows in the inset and the main panel).

$R$ as a function of temperature at different pressures. The data of $R$, normalized to the room-temperature value at zero pressure $R_{300, p=0}$, are displayed in Fig. 2 and split into three pressure ranges, similar to the specific heat data. Overall, the $T$-dependent behavior of $R / R_{300, p=0}$ is consistent with the literature at ambient pressure $[27,28,30]$. Initially, upon cooling, $R$ decreases, consistent with a semimetallic behavior [27] [see inset of Fig. 2(a)]. Below $\approx 80 \mathrm{~K}, R$ starts to increase rapidly [27]. Upon entering the magnetically ordered state at low temperatures, $R$ decreases due to loss of spin-disorder scattering, resulting in a peak of $R$ at the transition temperature. Over the entire pressure range up to $2.42 \mathrm{GPa}$, the peak value of $R$ increases with pressure with the strongest increase observed in the intermediate pressure range $1.4 \mathrm{GPa} \lesssim p \lesssim 1.95 \mathrm{GPa}$ 


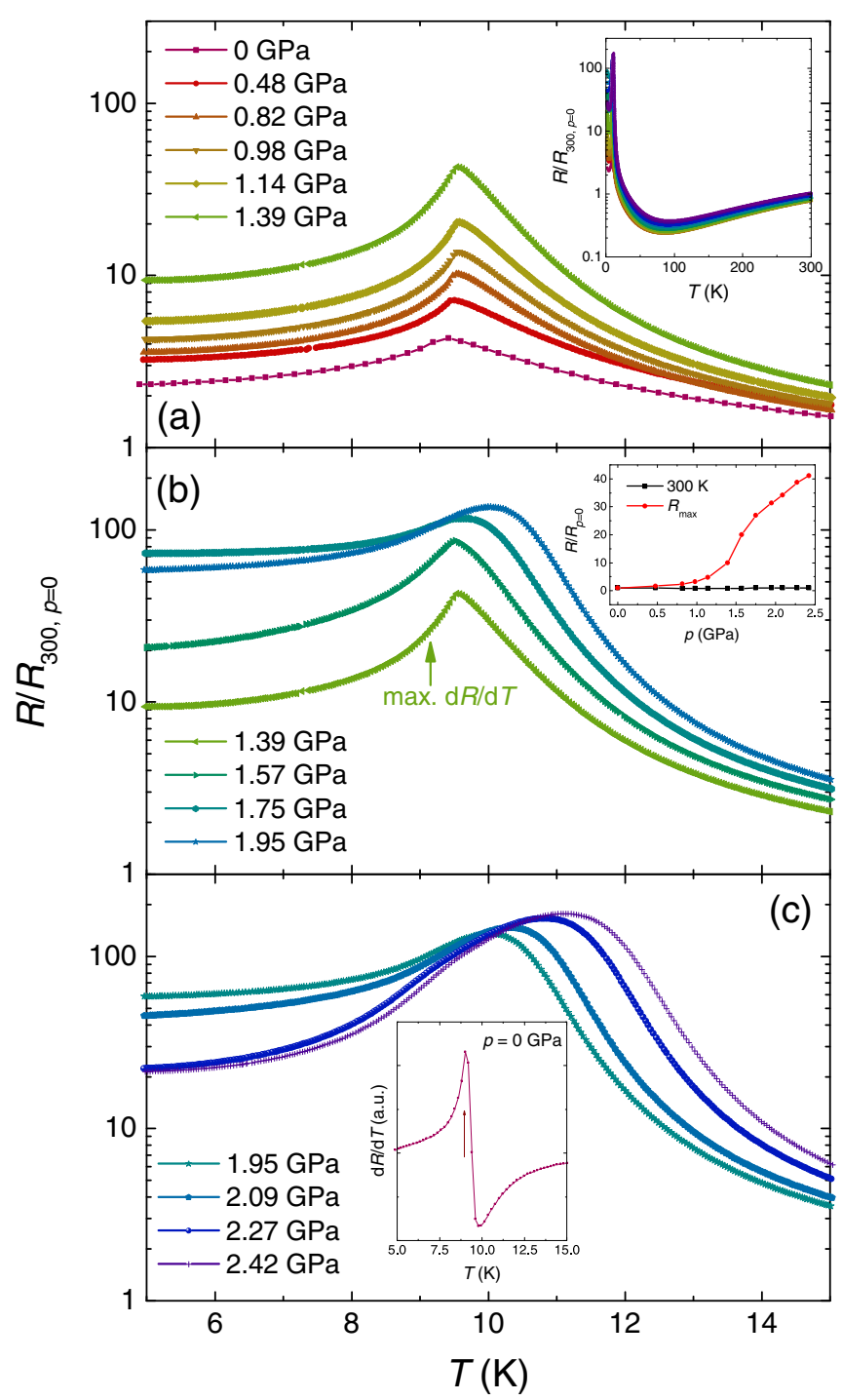

FIG. 2. Selected data sets of resistance, normalized to the zeropressure room-temperature value, $R / R_{300, p=0}$, vs $T(5 \mathrm{~K} \leqslant T \leqslant$ $15 \mathrm{~K}$ ) of $\mathrm{EuCd}_{2} \mathrm{As}_{2}$ under pressure $p$ for $0 \mathrm{GPa} \leqslant p \leqslant 1.39 \mathrm{GPa}$ (a), for $1.39 \mathrm{GPa} \leqslant p \leqslant 1.95 \mathrm{GPa}$ (b), and for $1.95 \mathrm{GPa} \leqslant p \leqslant$ $2.42 \mathrm{GPa}$ (c). Current was applied within the $a b$ plane. The inset in (a) shows all data over the full temperature range up to $300 \mathrm{~K}$. The arrows in (b) indicate the criterion which is used to determine the transition temperature (see text). The inset in (b) depicts the pressure dependence of $R$, normalized to its zero-pressure value $R_{p=0}$, at $T=300 \mathrm{~K}$ as well as of $R_{\max }$. The inset in (c) shows an example data set of $d R / d T$ at zero pressure, which is used to determine the transition temperatures (see arrow and text).

[see inset of Fig. 2(b)], while the room-temperature value of $R$ changes by less than $25 \%$. Similar to the specific heat data, we find that the peak in $R$ remains sharp for $p \lesssim 1.4 \mathrm{GPa}$ [Fig. 2(a)], starts to broaden for intermediate $p$ [Fig. 2(b)], and remains broad while clearly shifting to higher temperatures beyond $1.95 \mathrm{GPa}$ [Fig. 2(c)].

In order to construct the temperature-pressure phase diagram of $\mathrm{EuCd}_{2} \mathrm{As}_{2}$ from the data above, we used the following criteria. For the specific heat data, we determined the position of the minimum in $d(C / T) / d T$, as exemplarily shown in the

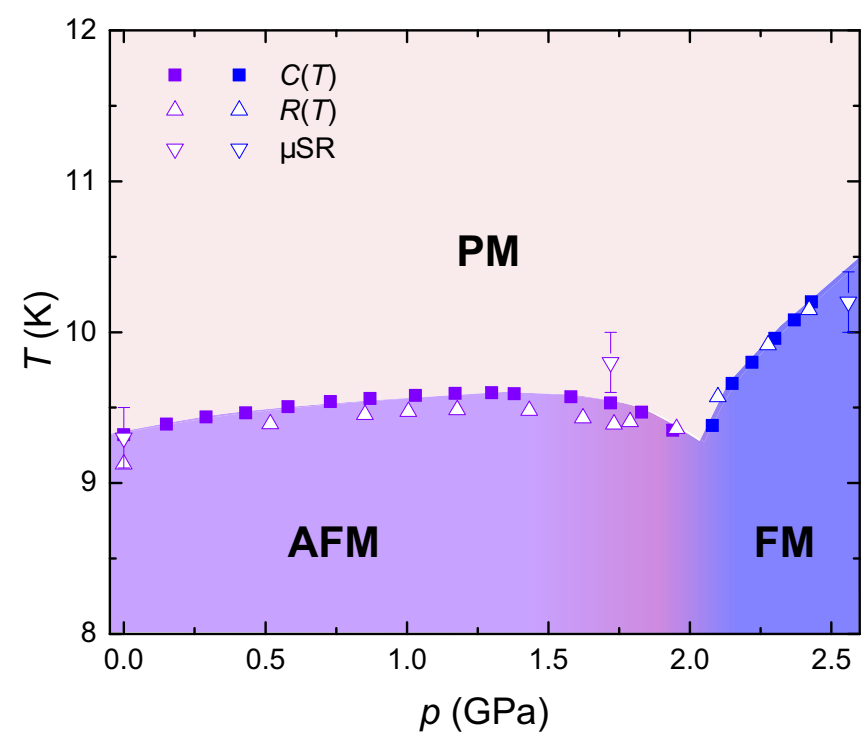

FIG. 3. Temperature-pressure (T-p) phase diagram of $\mathrm{EuCd}_{2} \mathrm{As}_{2}$, determined from specific heat, resistance, and $\mu \mathrm{SR}$ measurements. Light purple symbols correspond to the transition from the paramagnetic (PM) to the antiferromagnetic (AFM) state. Blue symbols indicate the position of the PM-ferromagnetic (FM) transition. The color shading in the intermediate pressure range indicates that $\mathrm{EuCd}_{2} \mathrm{As}_{2}$ shows strong tendencies towards $\mathrm{FM}$ order (see text for more details).

inset of Fig. 1(b). This criterion is close to the one obtained in isentropic constructions [see arrow in the main panel of Fig. 1(b)]. For the resistance measurements, we refer to the Fisher-Langer relation [55] for magnetic transitions in metals and chose the maximum of $d R / d T$ [see arrow in Fig. 2(b) and inset in Fig. 2(c)].

The resulting phase diagram is presented in Fig. 3. Pressure-dependent transition temperature data from both $C(T)$ and $R(T)$ runs agree with each other well and show an initial slow increase in $T_{\mathrm{N}}(p \lesssim 1.30 \mathrm{GPa})$ with pressure followed by a gradual decrease of $T_{\mathrm{N}}$ with further increase in pressure $(1.30 \mathrm{GPa} \lesssim p \lesssim 2.0 \mathrm{GPa})$. For $p \gtrsim 2.0 \mathrm{GPa}$ we observe a comparatively sharp increase in the magnetic ordering temperature. This sharp change in the pressure dependence of the ordering temperature strongly suggests that a different magnetic phase has been stabilized above the critical pressure $p^{\star} \simeq 2 \mathrm{GPa}$. Indeed, our $\mu \mathrm{SR}$ data, shown and discussed below, provide the basis for our determination that the high pressure phase has a ferromagnetic (FM) component to it.

\section{B. Microscopic study of magnetism under pressure}

To probe the magnetic properties under pressure, we used $\mu \mathrm{SR}$ measurements. In these experiments, approximately half of the muons stop in the sample and half in the pressure cell wall. The latter muons are sensitive to the magnetic fields that are generated by the sample inside the pressure cell. Thus, the signal from the muons stopping in the pressure cell wall will be measurably distorted [44,56,57] for ferromagnetic samples. A detailed description of the analysis procedure of the $\mu \mathrm{SR}$ data under hydrostatic pressure can be found in Appendix A 1 a. 


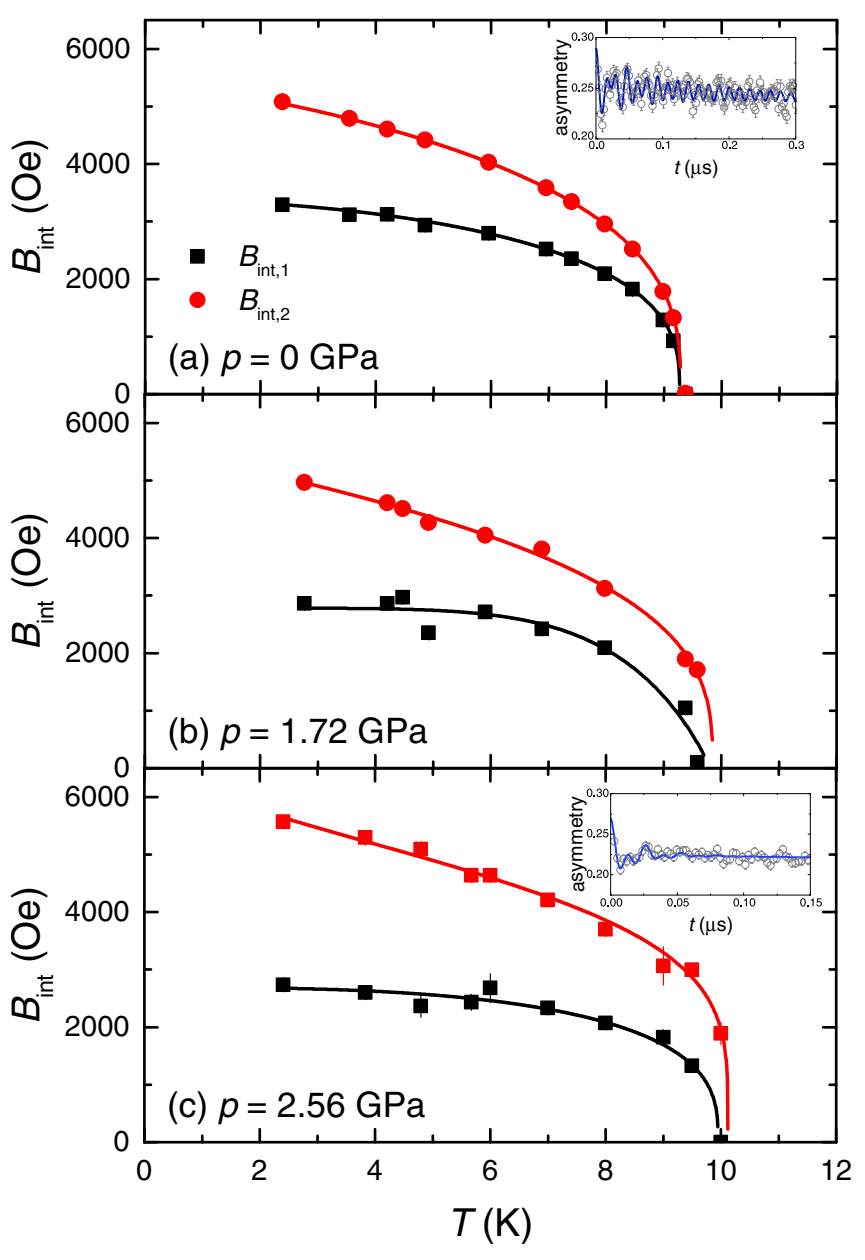

FIG. 4. Evolution of the internal field of the two stopping sites in $\mathrm{EuCd}_{2} \mathrm{As}_{2}, B_{\text {int, } 1}$, and $B_{\text {int, } 2}$ with temperature $T$ for pressures $p$ of (a) $0 \mathrm{GPa}$, (b) $1.72 \mathrm{GPa}$, and (c) $2.56 \mathrm{GPa}$. Note that the $0 \mathrm{GPa}$ data were taken inside the hydrostatic pressure cell. Lines represent fits to the empirical formula, $B_{\mathrm{int}, \mathrm{i}}=B_{\mathrm{int}, \mathrm{i}}^{0}\left[1-\left(\frac{T}{T^{\star}}\right)^{\alpha}\right]^{\beta}$ (see text). The insets in (a) and (b) show zero-field spectra (open gray symbols) taken at $T=3.55 \mathrm{~K}$ and $2.41 \mathrm{~K}$, respectively, together with the fit for two stopping sites (blue lines).

First, we present in Fig. 4 the evolution of the internal field of $\mathrm{EuCd}_{2} \mathrm{As}_{2}$ with temperature and pressure, which can be inferred from the zero-field $\mu \mathrm{SR}$ spectra [see insets in (a) and (c) for the asymmetry data at $0 \mathrm{GPa}$ and $2.56 \mathrm{GPa}$. In total, we took high-statistics zero field data sets for $0 \mathrm{GPa}$ [Fig. 4(a)], $1.72 \mathrm{GPa}$ [Fig. 4(b)], and $2.56 \mathrm{GPa}$ [Fig. 4(c)]. For all pressures, clear oscillations were observed below the respective pressure-dependent ordering temperature, signaling the presence of a finite internal field below the respective transition temperature. In each case, our data is best modeled by using two muon stopping sites with internal fields $B_{\text {int, } 1}$ and $B_{\text {int, } 2 \text {. }}$ For the $0 \mathrm{GPa}$ data, the best fit was obtained by using a ratio of 60:40 for the two stopping sites, whereas for higher pressures we fixed the ratio to 50:50. The transition temperatures from $\mu \mathrm{SR}$, which are included in the phase diagram in Fig. 3, were determined by fitting the $B_{\text {int,i }}$ data with the empirical formula $B_{\text {int, }}^{0}\left[1-\left(\frac{T}{T^{\star}}\right)^{\alpha}\right]^{\beta}$, with $B_{\text {int,i }}^{0}, T^{\star}, \alpha$, and $\beta$ being free parameters $\left(T^{\star}\right.$ corresponds to the respective transition temperature). Interestingly, the ratio of internal fields $B_{\text {int, } 2} / B_{\text {int, } 1}$ at $T \approx 2 \mathrm{~K}$

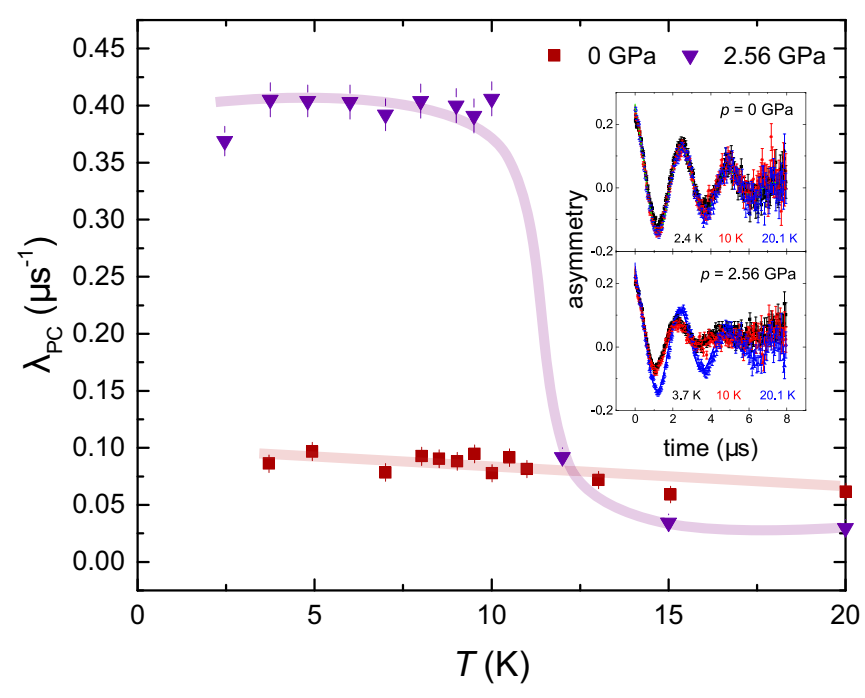

FIG. 5. Temperature dependence of the relaxation rate of the pressure cell $\lambda_{\mathrm{PC}}$ for pressures of $0 \mathrm{GPa}$ and $2.56 \mathrm{GPa}$. Lines are guide to the eyes. The insets show the weak-transverse field spectra at $0 \mathrm{GPa}$ and at $2.56 \mathrm{GPa}$ for temperatures below and above the phase transition.

increases significantly with pressure from 1.5 at $0 \mathrm{GPa}$ to 1.7 at $1.72 \mathrm{GPa}$ to 2 at $2.56 \mathrm{GPa}$. Even though we lack precise information on the muon stopping sites in $\mathrm{EuCd}_{2} \mathrm{As}_{2}$, the change of the ratio of internal fields suggests that there is some change of the magnetic structure, for example by rotation of the moments at a muon stopping site, with pressure [58].

Next, we discuss our results of the type of magnetic order under pressure. To this end, we show in Fig. 5 the pressure cell relaxation rate $\lambda_{\mathrm{PC}}$ at two different pressures, which were obtained from measuring the muon asymmetry in a weak transverse field of 30 Oe. As already evident from the raw data shown in the insets, there is no additional depolarization below the ordering temperature $T_{\mathrm{N}} \approx 9.5 \mathrm{~K}$ at ambient pressure $\left(p<p^{\star}\right)$, whereas there is a clear additional depolarization below $T_{\mathrm{C}} \approx 10.4 \mathrm{~K}$ at $2.56 \mathrm{GPa}\left(p>p^{\star}\right)$. As depicted in the main panel, the former data correspond to a weak temperature dependence of $\lambda_{\mathrm{PC}}$, the size of which equals the known relaxation rate of the pressure cell [44] (see Appendix A 1 a). This behavior is expected for antiferromagnetic order (see also our discussion of ambient-pressure $\mu \mathrm{SR}$ data on oriented single crystals in Appendix A $1 \mathrm{c}$ ). In contrast, for $2.56 \mathrm{GPa}, \lambda_{\mathrm{PC}}$ suddenly increases below $T_{\mathrm{C}}$ and levels off for lower temperatures, which can only be attributed to the presence of an additional field created by the sample. Importantly, this central result of our work represents compelling evidence for the realization of a FM state at high pressures. This conclusion is further supported by an increase of ferromagnetic fluctuations above the ordering temperature with pressure (see Appendix A 1 b). Based on the presented $\mu \mathrm{SR}$ data on an aggregate of randomly oriented single crystals, we cannot make any statement on whether the FM state is fully polarized along a crystallographic direction or whether there is only a ferromagnetic component to the order. Below, based on anisotropic magnetoresistance data, we argue that the high-pressure FM state is likely almost 

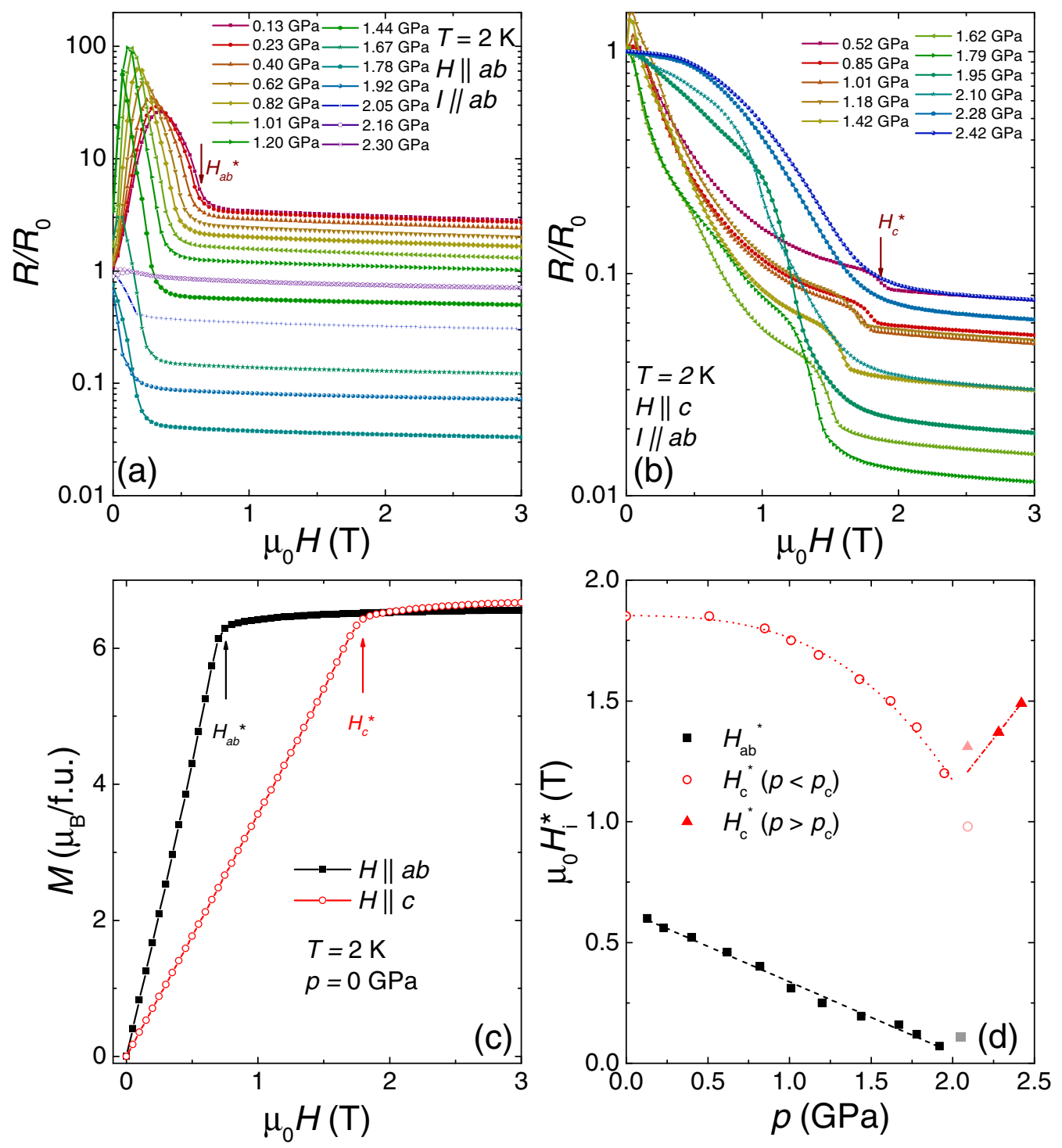

FIG. 6. Anisotropic magnetoresistance of $\mathrm{EuCd}_{2} \mathrm{As}_{2}$ at $T=2 \mathrm{~K}$ at different pressures. (a) In-plane $R / R_{0}$, with $R_{0}$ being the resistance in zero external field, as a function of in-plane field $\mu_{0} H$ at different pressures $p$. Arrow indicates the criterion to determine $H_{a b}^{\star}$. Note that the data at $2.16 \mathrm{GPa}$ and $2.30 \mathrm{GPa}$ lie on top of each other on this scale. (b) In-plane $R / R_{0}$ as a function of out-of-plane field $\mu_{0} H$ at different pressures $p$. Arrow indicates the criterion to determine $H_{c}^{\star}$. The features at very low field in this data set are likely a small in-plane component that is present due to slight misalignments of the sample with respect to the field. (c) Ambient-pressure anisotropic magnetization as a function of in-plane as well as out-of-plane magnetic field [33]. Arrows indicate the saturation fields $H_{a b}^{\star}$ and $H_{c}^{\star}$. (d) Evolution of $H_{a b}^{\star}$ and $H_{c}^{\star}$ with pressure. For $p<p_{c}$, the ground state is AFM with moments lying in the $a b$ plane. Thus, $H_{a b}^{\star}$ and $H_{c}^{\star}$ are defined as saturation fields into fully polarized states with moments in the $a b$ and $c$ direction, respectively. For $p>p_{c}$, the ground state is FM with moments in the $a b$ plane, thus only $H_{c}^{\star}$ can be defined. The light data points in the immediate vicinity of $p_{c}$ are discussed in Sec. A 2 .

in-plane polarized, and thus we refer here to the notion of FM order.

\section{Anisotropic magnetoresistance data}

Now that we have established a change of the magnetic ground state from AFM to FM by hydrostatic pressure in $\mathrm{EuCd}_{2} \mathrm{As}_{2}$, we want to discuss transport measurements under pressure in finite magnetic field in the ordered states. The aim of these experiments is to identify the moment direction in the high-pressure state.
In Figs. 6(a) and 6(b), we show our data of the pressuredependent magnetoresistance (MR) data at $T=2 \mathrm{~K}$, taken in a longitudinal and in a transverse configuration, i.e., $\mu_{0} H\|I\| a b$, (a) and $\mu_{0} H\|c, I\| a b$ (b). At lowest pressures ( $0.13 \mathrm{GPa}$ and $0.52 \mathrm{GPa}$, respectively), the longitudinal $R / R_{0}$ goes through a maximum and becomes almost field independent above $\mu_{0} H_{a b}^{\star} \sim 0.7 \mathrm{~T}$ [Fig. 6(a)], whereas the transverse $R / R_{0}$ shows a steplike change around $\mu_{0} H_{c}^{\star} \sim$ $1.8 \mathrm{~T}$ [Figs. 6(c) and 6(d)]. Those field scales coincide very well with the saturation fields for the specific field orientations, determined from magnetization measurements at ambient pressure [33] [see Fig. 6(c)]. The specific 
criteria, which were used to determine $\mu_{0} H_{a b}^{\star}$ and $\mu_{0} H_{c}^{\star}$ from the low-pressure and high-pressure data sets, are discussed in Sec. A 2.

As clearly visible in the raw data [Fig. 6(a)], $H_{a b}^{\star}$ decreases rapidly with increasing pressure. As shown in Fig. 6(d), $H_{a b}^{\star}$ extrapolates to zero at $\approx 2 \mathrm{GPa}$, i.e., at $p_{c}$ (the behavior of the data at $2.05 \mathrm{GPa}$ is discussed in Sec. A 2). For $2.3 \mathrm{GPa}$, the $R / R_{0}$ data is essentially field independent. These results suggest that the high-pressure FM state has its moments aligned in the plane, i.e., a pure FM state: With pressure, we suppress $H_{a b}^{\star}$ to zero, implying full moment alignment along the in-plane direction.

Whereas $H_{a b}^{\star}$ is suppressed to zero with increasing pressure, $H_{c}^{\star}$ is also suppressed but remains finite at $2 \mathrm{GPa}$. For $p \gtrsim 2 \mathrm{GPa}$, no steplike change can be observed. Instead, we observe a broad crossover feature in the field-dependent $R / R_{0}$ data, the position of which increases with increasing pressure [see Fig. 6(d)]. The absence of a metamagnetic transition for out-of-plane fields is consistent with the notion of FM order with moments oriented in the $a b$ plane. The decrease in $H_{c}^{\star}$ for $p<p_{c}$ is nonetheless remarkable as it suggests that the energy difference to the FM state with moments along the $c$ axis is also reduced with pressure. We will further discuss the proximity to this $\mathrm{FM}_{c}$ state based on DFT calculations below. We note that our Hall data under pressure, presented in Appendix A 3, indicate the possibility that the change of magnetic structure across $p_{c}$ is associated with a change of charge carrier density.

\section{Results of DFT calculations under pressure}

In this section we study the pressure-dependent evolution of magnetic order in $\mathrm{EuCd}_{2} \mathrm{As}_{2}$ and the possible transition from in-plane AFM (denoted by $\mathrm{AFM}_{a b}$ in the following) to in-plane FM order (denoted by $\mathrm{FM}_{a b}$ ) for low pressures with the help of DFT calculations. To this end, we constructed the equation of state (EOS) for both $\mathrm{AFM}_{a b}$ and $\mathrm{FM}_{a b}$ spin configurations around the equilibrium volume and fitted it to the Birch-Murnaghan equation [59,60]. The optimized equilibrium lattice constants using the PBE exchange-correlation functional and $U=4.4 \mathrm{eV}$ without spin-orbit coupling (SOC) for A-type antiferromagnetic (i.e., $\mathrm{AFM}_{a b}$ ) spin configuration are $a=4.49 \AA$ and $c=7.37 \AA$. These values agree with the experimental data of 4.4336 and $7.2925 \AA$ at $T=11 \mathrm{~K}$, respectively, just above $T_{N}$ (see Appendix A 4) within about $1 \%$, which can be considered as a very good agreement for DFTPBE calculations [61]. For EOS calculations, at each volume, the cell shape and atomic positions are fully relaxed for both $\mathrm{AFM}_{a b}$ and $\mathrm{FM}_{a b}$ with SOC, with the magnetic moment pointing in the in-plane direction to the nearest-neighbor (NN) Eu or equivalently along the $a$ or $b$ axis. The fitted equilibrium volumes from EOS are 129.78 and $129.76 \AA^{3}$ /f.u. for $\mathrm{AFM}_{a b}$ and $\mathrm{FM}_{a b}$ with SOC, respectively, having an energy difference of $0.3 \mathrm{meV} /$ f.u. in favor of the $\mathrm{AFM}_{a b}$ state. This agrees well with the $0.22 \mathrm{meV} /$ f.u. preference to $\mathrm{AFM}_{a b}$ from the direct DFT calculation at the initial AFM equilibrium volume of $128.96 \AA^{3} /$ f.u. With such small differences in equilibrium volume and energy, the two fully-relaxed EOS of the competing magnetic phases are aligned very closely together to have almost the same bulk modulus of $46.7 \mathrm{GPa}$. To obtain the

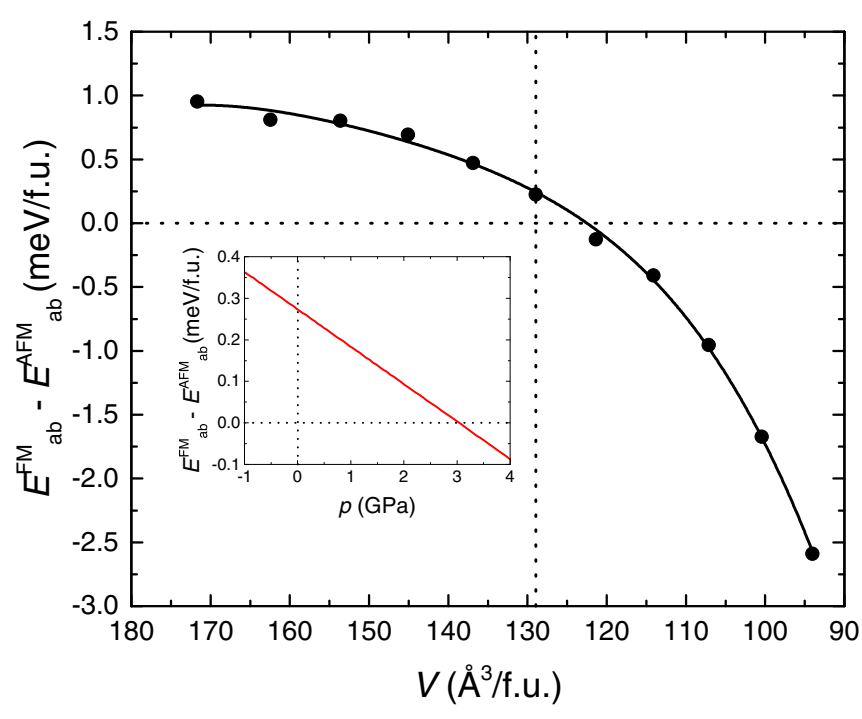

FIG. 7. Equation of state (EOS) energy difference $\left(\Delta E_{\mathrm{FM}, a b-\mathrm{AFM}, a b}\right)$ vs volume $(V)$ between the fully relaxed FM and A-type AFM, where in both cases the moment point is in-plane to the nearest-neighbor $\mathrm{Eu}$. The initial $\mathrm{AFM}_{a b}$ equilibrium volume of $128.96 \AA^{3}$ /f.u. as well as the phase transition at $\Delta E_{\mathrm{FM}, a b-\mathrm{AFM}, a b}=0.0$ are indicated by the vertical and horizontal dashed line, respectively. The inset shows an enlarged view around the $\mathrm{AFM}_{a b}-\mathrm{FM}_{a b}$ transition region with the $x$ axis converted to pressure ( $p$ ) using the calculated bulk modulus (see text). The dashed line for $\Delta E_{\mathrm{FM}, a b-\mathrm{AFM}, a b}=0.0$ gives the critical pressure $p_{c} \approx 3.0 \mathrm{GPa}$.

critical pressure $p_{c}$ for the AFM-FM magnetic phase transition, the difference between the two EOS, $\Delta E_{\mathrm{FM}, a b-\mathrm{AFM}, a b}$, vs volume is plotted in Fig. 7. At the initial $\mathrm{AFM}_{a b}$ equilibrium volume, $\mathrm{AFM}_{a b}$ is preferred by $0.22 \mathrm{meV} /$ f.u. This preference is increased at larger volume but decreased for smaller volume, i.e., with increasing hydrostatic pressure. This leads to a $\mathrm{AFM}_{a b}-\mathrm{FM}_{a b}$ magnetic phase transition under hydrostatic pressure, as observed in experiment. The transition region is shown on enlarged scales in the inset of Fig. 7 with the $x$ axis converted to pressure using the calculated bulk modulus. The dashed line of $\Delta E_{\mathrm{FM}, a b-\mathrm{AFM}, a b}=0.0$ gives the critical pressure $p_{c} \approx 3.0 \mathrm{GPa}$, in good agreement with the experimental data of $\approx 2.0 \mathrm{GPa}$. To firmly establish the pressure-induced $\mathrm{AFM}_{a b}-\mathrm{FM}_{a b}$ in $\mathrm{EuCd}_{2} \mathrm{As}_{2}$ within DFT, we have also tested different exchange-correlation functionals, PAW potentials, and computational settings, including using the same relaxed crystal structures for $\mathrm{AFM}_{a b}$ and $\mathrm{FM}_{a b}$ at each volume from either the nonmagnetic or $\mathrm{AFM}_{a b}$ configuration. All calculations confirm a $\mathrm{AFM}_{a b}-\mathrm{FM}_{a b}$, with $p_{c}$ values in the range from 2.7 to $4.2 \mathrm{GPa}$ (see Appendix A 5 for a detailed discussion on different initial conditions).

Next, we calculated the crystalline magnetoanisotropy energy (MAE), defined by the required energy to switch the crystal magnetization from the low pressure basal plane magnetic order and direction to, in this case explicitly, $\mathrm{FM}_{c}$ order at increasing pressure. Such a calculation provides information about the proximity of $\mathrm{EuCd}_{2} \mathrm{As}_{2}$ to the $\mathrm{FM}_{c}$ state, which, according to recent work, is able to host a single pair of Weyl nodes $[24,25]$. This calculation was done in various steps. First, we relaxed the unit cell of $\mathrm{EuCd}_{2} \mathrm{As}_{2}$ at different 


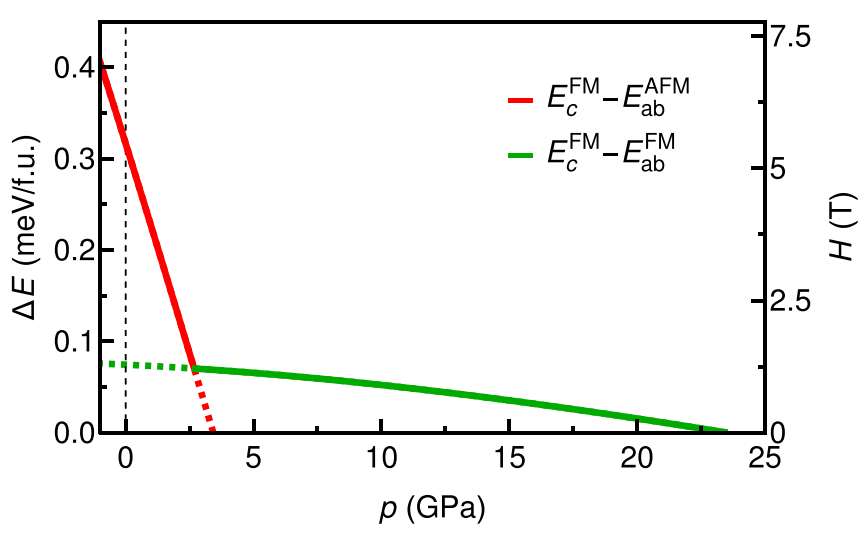

FIG. 8. Energy difference of the $\mathrm{FM}_{c}$ order to the ground states (left axis) and corresponding magnetic field (right axis) vs pressure. The solid lines show the energy difference between the $\mathrm{FM}_{c}$ state and the respective ground states, i.e., $\mathrm{AFM}_{a b}$ for $p \lesssim 3 \mathrm{GPa}$ (red) and $\mathrm{FM}_{a b}$ for $p \gtrsim 3 \mathrm{GPa}$ (green). Dashed lines represent the energy differences between non-ground-state magnetic configurations. The ground state transition to $\mathrm{FM}_{c}$ takes place at approximately $p_{c}^{\prime} \approx$ $23 \mathrm{GPa}$.

volumes by treating the $\mathrm{Eu}^{2+} 4 f$ states completely as core states and by performing non-spin-polarized calculations with the PBE exchange correlation functional. The former warrants that no spurious Eu $f$ states appear near the Fermi level and the latter ensures a fully nonmagnetic setting since no magnetic moments/spins are invoked in the calculation. Such a relaxation guarantees a uniform change in the lattice without being influenced by the nature of the assumed magnetism for the $\mathrm{Eu}^{2+} 4 f$ states. The resulting optimized nonmagnetic equilibrium lattice parameters are given by $a=4.48 \AA$ and $c=7.27 \AA$, which are closer to the experimental data (see Appendix A 4) than the magnetically relaxed structures, due to the absence of long-range magnetic order at the measured $T=$ $11 \mathrm{~K}$. In a second step, we took the optimized nonmagnetic crystal structures and calculated total energies for different Eu magnetic configurations, by including the $\mathrm{Eu}^{2+} 4 f$ states as valence electrons with $U=4.4 \mathrm{eV}$ and SOC and with the Eu moments aligned either antiferromagnetically or ferromagnetically and moments pointing in the plane for $\mathrm{AFM}_{a b}$ and $\mathrm{FM}_{a b}$, respectively, as well as aligned ferromagnetically along the $c$ axis, denoted as $\mathrm{FM}_{c}$. The DFT results were fitted to the Birch-Murnagham EOS, yielding the MAE by subtracting the corresponding fits. After fitting, the equilibrium volume increases from $126.4 \AA^{3} /$ f.u. for the nonmagnetic configuration to $130.21 \AA^{3} /$ f.u. for both FM orders and to $130.21 \AA^{3} /$ f.u. for $\mathrm{AFM}_{a b}$. Note that throughout all performed calculations we keep the valence of Eu fixed to 2+, so that we cannot exclude the possibility of a valence transition $\left(\mathrm{Eu}^{2+}\right.$ to $\left.\mathrm{Eu}^{3+}\right)$ at the simulated higher pressures. This goes beyond the scope of the present work.

In Fig. 8 we show the results of these MAE calculations as a function of hydrostatic pressure. The MAE is directly proportional to the $H_{c}^{\star}$ field that is measured in experiment. At ambient pressure, the theoretical calculations overestimate the experimental $H_{c}^{\star}$ value, which is expected in the framework of DFT and Birch-Munagham EOS, which we use. Nevertheless, the tendencies as a function of pressure are robust. Here, we find that the MAE or $H_{c}^{\star}$ decrease rapidly with increasing pressure for $p<p_{c}$, consistent with our experimental observations, shown in Fig. 6(d). Above $p_{c}$, the MAE still continues to decrease with increasing pressure, however at a slower rate. This trend is opposite to what is observed for our experimental data for pressures close to $p_{c}$. However, we note that the details of our theoretical prediction close to $p_{c}$ depend on the details of the relaxation (the result of a different relaxation is shown in Fig. 15). Thus, experimental data to higher pressures is needed for a meaningful comparison between theory and experiment in the $\mathrm{FM}_{a b}$ ground state region.

The decrease of MAE with pressures beyond $\approx 10 \mathrm{GPa}$ does not depend on details of the relaxation. Thus, there will eventually be a transition to the $\mathrm{FM}_{c}$ state at sufficiently high pressures, as long as Eu does not change its valency. We predict that this transition to an $\mathrm{FM}_{c}$ state occurs at $p_{c}^{\prime} \approx 23 \mathrm{GPa}$ Whereas this pressure is larger than the experimentally used pressures in this work, it is nevertheless experimentally feasible to realize those pressures. Thus, based on our theoretical calculations, it might be possible to stabilize the desired $\mathrm{FM}_{c}$ state in $\mathrm{EuCd}_{2} \mathrm{As}_{2}$ in experiment as a result of pressure effects and without application of an external magnetic field and to study key transport quantities with respect to topology. At the same time, it will be very interesting to perform further studies to understand the microscopic origin of the high tunability of the magnetic properties of $\mathrm{EuCd}_{2} \mathrm{As}_{2}$.

\section{CONCLUSIONS}

In summary, we presented an experimental study of the temperature-pressure phase diagram up to $\sim 2.5 \mathrm{GPa}$ of the magnetic Weyl semimetal $\mathrm{EuCd}_{2} \mathrm{As}_{2}$ by means of specific heat, transport, and $\mu$ SR measurements and performed DFT calculations up to pressures of $\approx 25 \mathrm{GPa}$. Our results clearly show that this compound undergoes a transition from an antiferromagnetic state at low pressures to a ferromagnetic state above $2 \mathrm{GPa}$, in which moments are dominantly oriented within the $a b$ plane. In addition, we find that pressure also drives $\mathrm{EuCd}_{2} \mathrm{As}_{2}$ closer to the sought-for FM state, in which moments are oriented along the $c$ axis. We predict that a hydrostatic pressure of $\approx 23 \mathrm{GPa}$ will stabilize this $\mathrm{FM}_{c}$ state if no valence transition of $\mathrm{Eu}^{2+}$ intervenes. Overall, our study identifies a clear and experimentally accessible tuning route to change the magnetic ground state in $\mathrm{EuCd}_{2} \mathrm{As}_{2}$ in a single sample and investigate the interplay of magnetism and topological nontrivial phases. Our results motivate further experimental and theoretical studies on $\mathrm{EuCd}_{2} \mathrm{As}_{2}$ under pressure, which is a very powerful parameter to tune the properties of this magnetic Weyl semimetal.

\section{ACKNOWLEDGMENTS}

We thank Na Hyun Jo and Young-Joon Song for useful discussions. S.L.B. and P.C.C. thank B. Schweinsteiger for inspiring research group members. Work at the Ames Laboratory was supported by the U.S. Department of Energy, Office of Science, Basic Energy Sciences, Materials Sciences and Engineering Division. The Ames Laboratory is operated for the U.S. Department of Energy by Iowa State University under Contract No. DEAC02-07CH11358. E.G. and L.X. were 
funded, in part, by the Gordon and Betty Moore Foundation's EPiQS Initiative through Grant No. GBMF4411. B.K. and L.-L.W. were funded by the Center for Advancement of Topological Semimetals, an Energy Frontier Research Center funded by the U.S. Department of Energy Office of Science, Office of Basic Energy Sciences, through the Ames Laboratory under its Contract No. DE-AC02-07CH11358. Research of R.G. is supported by the Swiss National Science Foundation (SNF-Grant No. 200021-175935). A.V. and R.V. acknowledge support by the Deutsche Forschungsgemeinschaft (DFG, German Research Foundation) for funding through TRR 288 - 422213477 (Project No. B05).

\section{APPENDIX A: APPENDIX}

\section{Additional discussion of $\mu \mathrm{SR}$ data under pressure}

\section{a. Analysis of $\mu \mathrm{SR}$ data inside a pressure cell}

Zero-field data analysis. The zero-field $\mu$ SR data was analyzed by taking two independent contributions to the total asymmetry $A(t)$ into account

$$
A^{\mathrm{ZF}}(t)=A_{\mathrm{S}}^{\mathrm{ZF}}(t)+A_{\mathrm{PC}}^{\mathrm{ZF}}(t),
$$

with $A_{\mathrm{S}}^{\mathrm{ZF}}(t)\left[A_{\mathrm{PC}}^{\mathrm{ZF}}(t)\right]$ being the contribution from the sample (the pressure cell). The sample contribution for $\mathrm{EuCd}_{2} \mathrm{As}_{2}$ was found to be best described by considering two stopping sites with different internal field, resulting in

$$
\begin{aligned}
A_{\mathrm{S}}^{\mathrm{ZF}}(t)= & A_{\mathrm{S}, 0}^{\mathrm{ZF}}\left[\sum _ { i = 1 } ^ { 2 } m _ { i } \left(\frac{2}{3} \cos \left(\gamma_{\mathrm{m}} B_{\mathrm{int}, \mathrm{i}}\right) \exp \left(-\lambda_{\mathrm{T}, \mathrm{i}}\right)\right.\right. \\
& \left.+\frac{1}{3} \exp \left(-\lambda_{\mathrm{L}, \mathrm{i}}\right)\right) \\
& \left.+\left(1-m_{1}-m_{2}\right) \exp (-\Lambda t)\right]
\end{aligned}
$$

with $m_{i}$ the magnetic fraction of the $i$ th component, $\gamma_{\mathrm{m}}$ the gyromagnetic ratio of the muon, $B_{\mathrm{int,i}}$ the internal field, $\lambda_{\mathrm{T}, \mathrm{i}}$ the transverse relaxation rate, $\lambda_{\mathrm{L}, \mathrm{i}}$ the longitudinal relaxation rate, and $\Lambda$ the relaxation rate of the paramagnetic portion of the sample. The $2 / 3$ and $1 / 3$ components arise from averaging over a large aggregate of arbitrarily oriented single crystals. The background contribution from the pressure cell can be determined in an independent set of experiment and can be described by two depolarization channels (one originating from nuclear moments and one from electronic moments) following a damped Kubo-Toyabe form

$$
\begin{aligned}
A_{\mathrm{PC}}^{\mathrm{ZF}}(t)= & A_{\mathrm{PC}, 0}^{\mathrm{ZF}}\left(\frac{1}{3}+\frac{2}{3}\left(1-\sigma_{\mathrm{PC}}^{2} t^{2}\right)\right) \\
& \times \exp \left(-\sigma_{\mathrm{PC}}^{2} t^{2} / 2\right) \exp \left(-\lambda_{\mathrm{PC}} t\right),
\end{aligned}
$$

with $\sigma_{\mathrm{PC}}\left(\lambda_{\mathrm{PC}}\right)$ the relaxation rate associated with the nuclear (electronic) moments.

Weak transverse field data analysis. As mentioned in the main text, there exists an additional depolarization of the muons stopping in the pressure cell when the sample inside the cell exhibits a strong magnetization. We note that the muons which stop in the pressure cell wall mostly stop inside the inner cylinder, i.e., very close to the sample, so that they can be sensitive to stray fields emerging from the sample, as shown in recent calculations of the muon stopping profile

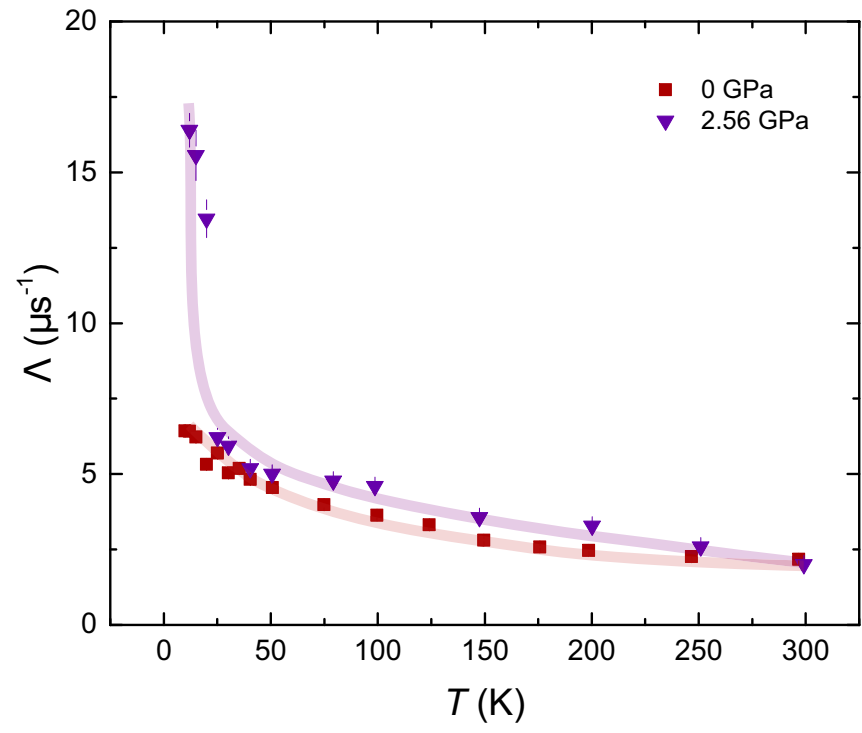

FIG. 9. Temperature dependence of the dynamical relaxation rate $\Lambda$ of $\mathrm{EuCd}_{2} \mathrm{As}_{2}$ for pressures of $0 \mathrm{GPa}$ and $2.56 \mathrm{GPa}$ for $10 \mathrm{~K}<T<300 \mathrm{~K}$ and $12 \mathrm{~K}<T<300 \mathrm{~K}$, respectively, i.e., in the paramagnetic state at each pressure. Lines are guide to the eyes.

for pressure-cell experiments with this particular muon energy [62]. In the case of weak-transverse field experiments with finite $B_{\mathrm{ex}}$, the pressure cell contribution thus reads as

$$
\begin{aligned}
A_{\mathrm{PC}}^{\mathrm{wTF}}(t)= & A_{\mathrm{PC}, 0}^{\mathrm{wTF}}(t) \exp \left(-\sigma_{\mathrm{PC}}^{2} t^{2} / 2\right) \\
& \times \exp \left(-\lambda_{\mathrm{PC}}\right) \cos \left(\gamma_{\mathrm{m}} B_{\mathrm{ex}} t+\phi\right) .
\end{aligned}
$$

Here, $\sigma_{\mathrm{PC}}$ refers to the relaxation rate caused by nuclear moments, whereas $\lambda_{\mathrm{PC}}$ is the relaxation rate determined by the electronic moments as well as the influence of the field that is created by a sample with macroscopic magnetization. The electronic contribution to $\lambda_{\mathrm{PC}}$ is typically almost temperature independent [44] and $\lambda_{\mathrm{PC}} \simeq 0.05-0.1 \mu \mathrm{s}$ (see also data above the ordering temperature in Fig. 5).

\section{b. Evolution of muSR relaxation above the ordering temperature with pressure}

We now discuss the relaxation rate $\Lambda$ of $\mathrm{EuCd}_{2} \mathrm{As}_{2}$ above the ordering temperature, as shown in Fig. 9. For ambient pressure, we find a moderate increase of $\Lambda$ with lowering temperature, which is fully consistent with the expectations for paramagnetism stemming from the large $\mathrm{Eu}^{2+}$ moments. Importantly, we note that we do not find any indications for the steplike change of $\Lambda$ at $\approx 100 \mathrm{~K}$, which Ma et al. [30] observed and which was taken as strong evidence for quasi-long-range and quasistatic magnetic order below $100 \mathrm{~K}$ that stabilizes Weyl physics even without spontaneous breaking of timereversal symmetry (see Appendix A $1 \mathrm{c}$ for supporting $\Lambda$ data on a well-oriented single crystal at ambient pressure outside the pressure cell). Instead, the large contribution from the $\mathrm{Eu}$ paramagnetism does not allow for any conclusion about the presence or nature of magnetic correlations. However, upon increasing pressure to $p=2.56 \mathrm{GPa}$, we find that $\Lambda$ increases much faster below $\approx 20 \mathrm{~K}$, reflecting a strong enhancement of FM fluctuations in the proximity of the FM ground state. 


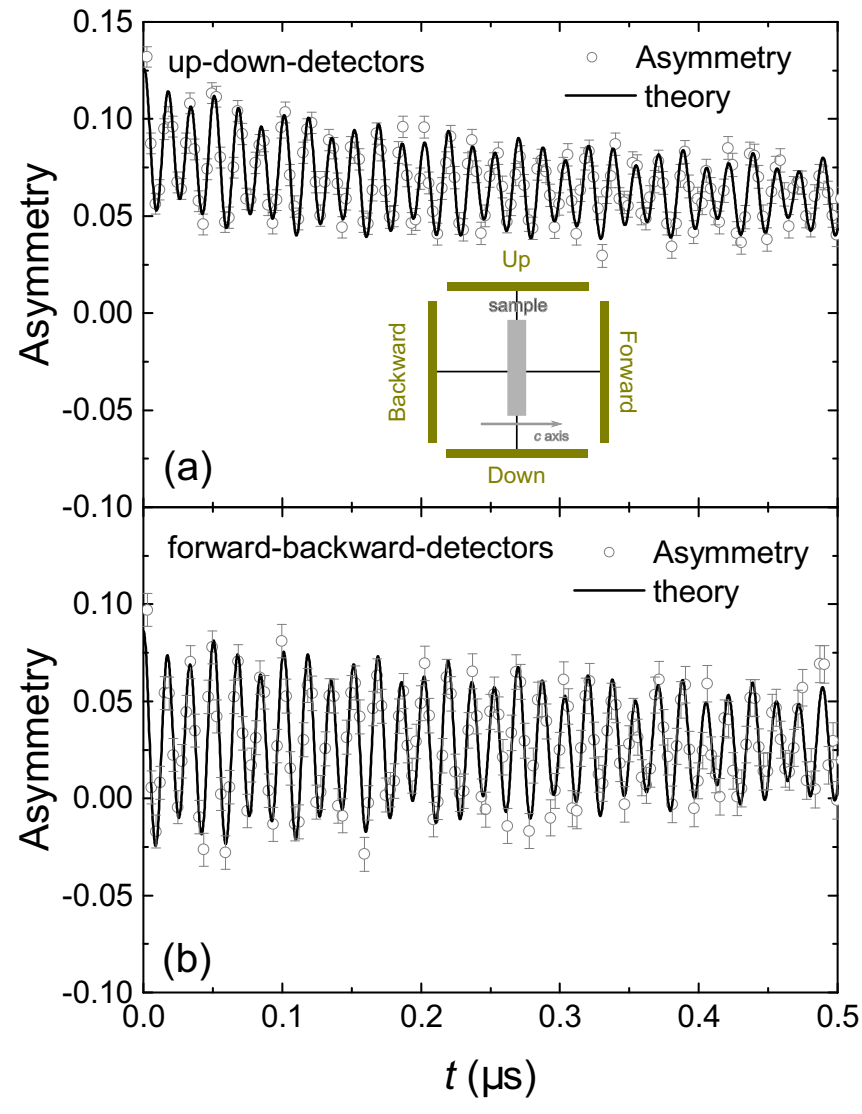

FIG. 10. Ambient-pressure zero-field $\mu$ SR asymmetry of an oriented single crystal of $\mathrm{EuCd}_{2} \mathrm{As}_{2}$ using the GPS spectrometer. The orientation of the sample with respect to the detectors is indicated in the inset of (a). The asymmetry is evaluated from the difference in counts of the up and down detectors (a) as well as the forward and backward detectors (b).

\section{c. $\mu \mathrm{SR}$ data at ambient pressure}

So as to characterize the magnetic order at ambient pressure in more detail, we performed $\mu \mathrm{SR}$ measurements in zero field on an oriented single crystal in the GPS spectrometer. Given that the sample is oriented, information on the orientation of the magnetic order can be inferred from differences in counts in the forward and backward vs the up and down detectors (see Fig. 10 for a schematic sketch of the experimental setup). Clear oscillations are seen in the asymmetry evaluated from the difference between the up and down detectors [Fig. 10(a)] as well as the forward and backward detectors [Fig. 10(b)]. This implies that the moments are not aligned out-of-plane at ambient pressure, consistent with earlier literature results $[28,29]$. The data is best described by taking two muon stopping sites into account and the respective internal fields inferred from these ambient-pressure measurements agree very well with the ones plotted in Fig. 4, which were inferred from the data inside the pressure cell at $0 \mathrm{GPa}$.

In Fig. 11, we show the relaxation rate $\Lambda$ above the ordering temperature inferred from the GPS experiment on the oriented single crystal and compare it with the data inferred from the randomly oriented aggregate of single crystals inside the pressure cell at ambient pressure. Whereas the overall

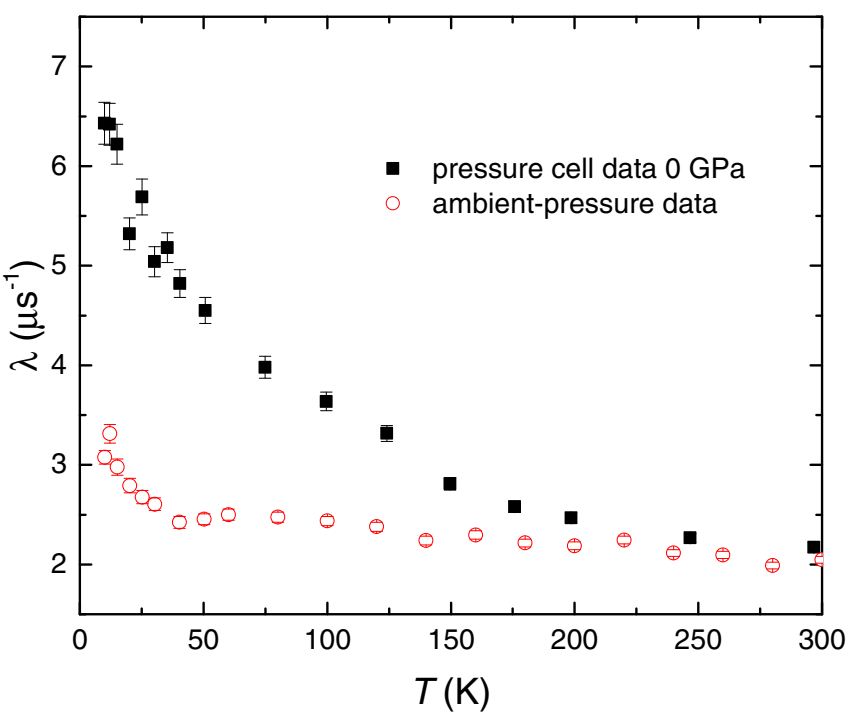

FIG. 11. Temperature dependence of the dynamical relaxation rate $\Lambda$ of $\mathrm{EuCd}_{2} \mathrm{As}_{2}$ above the magnetic ordering temperature. Data were taken inside the pressure cell at ambient pressure on a arbitrarily oriented aggregate of single crystals (black symbols) as well as an oriented single crystal at ambient pressure outside of the pressure cell (red symbols).

magnitude of $\Lambda$ differs between the two experiments, which likely can be attributed to a directional-dependent size of the relaxation, none of the data reveal a clear feature at $\approx 100 \mathrm{~K}$, as previously stated in Ref. [30].

\section{Criteria to determine $H_{a b}^{\star}$ and $H_{c}^{\star}$ from pressure-dependent magnetoresistance data}

Here we outline the choice of criteria for the determination of $H_{a b}^{\star}$ and $H_{c}^{\star}$ as a function of pressure. To this end, we refer to the derivative of $\log \left(R / R_{0}\right)$ with respect to field, $\mu_{0} H$, for the two field orientations (see Fig. 12). For field applied along the in-plane direction [Fig. 12(a)], a clear minimum is seen for
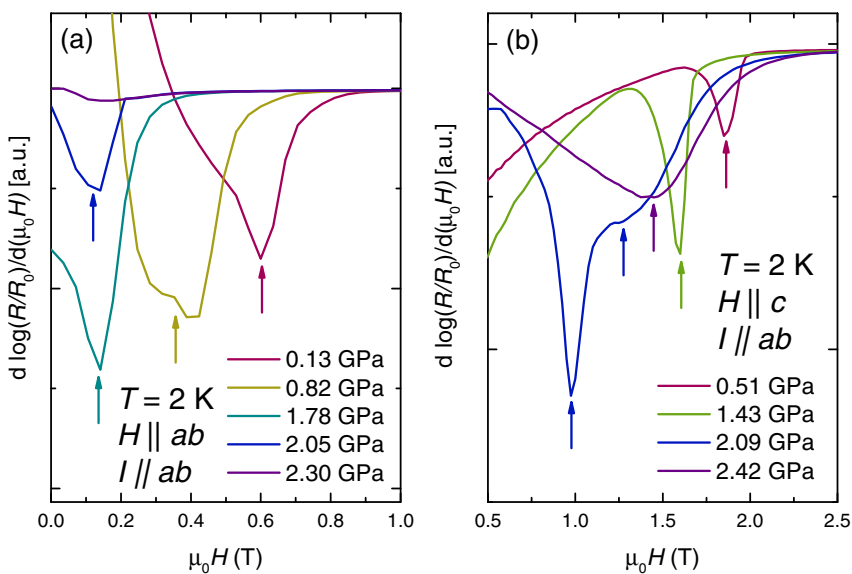

FIG. 12. Derivative of $\log \left(R / R_{0}\right)$ with respect to field, $\mu_{0} H$, for the field oriented along the in-plane direction (a) and out-of-plane direction (b) for selected pressures. The arrows indicate the position of minima that are chosen as criteria for the determination of $H_{a b}^{\star}$ and $H_{c}^{\star}$. 

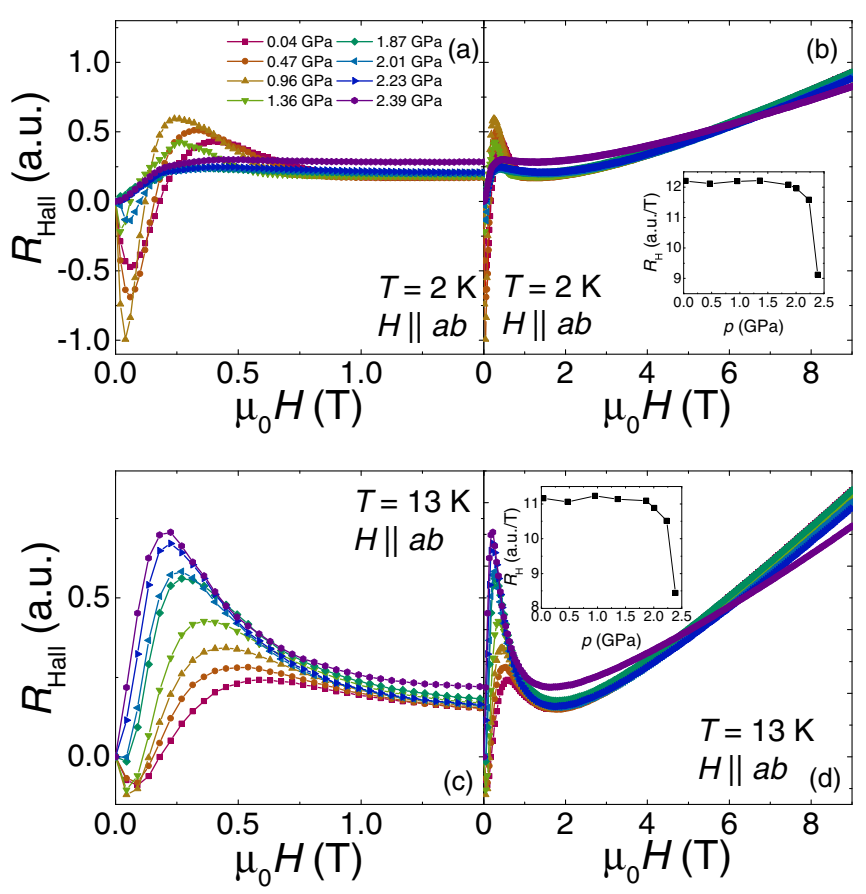

FIG. 13. Hall resistance $R_{\text {Hall }}$ of $\mathrm{EuCd}_{2} \mathrm{As}_{2}$ at different pressures at $T=2 \mathrm{~K}$ (a),(b) and $T=13 \mathrm{~K}$ (c),(d) in low fields (a),(c) and high fields (b),(d). Data were taken with magnetic field along the in-plane direction. The insets show the high-field slope of the Hall data as a function of pressure at the respective temperatures.

$p<p_{c} \sim 2 \mathrm{GPa}$, the position of which we use to determine $H_{a b}^{\star}$. For $p>p_{c}$ (represented by the $2.30 \mathrm{GPa}$ data), no clear feature is resolved for $\mu_{0} H \| a b$. A clear minimum for $p<p_{c}$ can also be observed for field along the out-of-plane direction [Fig. 12(b)], which we use to determine $H_{c}^{\star}\left(p<p_{c}\right)$. For $p>p_{c}$ we find a broad minimum, reflecting the crossover from moment orientation in the $a b$ plane to the $c$ axis. We refer to the position of the broad minimum as $H_{c}^{\star}\left(p>p_{c}\right)$. We note that for pressures close to $p_{c}$ the data in (b) reveal a sharp feature as well as a broad minimum, manifesting features of both the low- and high-pressure phase. Thus, we include two data points in Fig. 6 for $H_{c}^{\star}$. In addition, the $2.05 \mathrm{GPa}$ data set for $\mu_{0} H \| a b$ still shows a minimum, however much reduced in size compared to the low-pressure data. At present, we can only speculate about the detailed magnetic structure close to $p_{c}$. It might be that phase separation occurs close to $p_{c}$ or canting of the moments is increasingly important close to the pressure-induced phase transition.

\section{Hall effect measurements under pressure}

Hall effect measurements were made by carefully painting two current contacts to cover the two opposite ends of the crystal to ensure as uniform of a current density as possible and two voltage contact on the remaining two side surfaces of the crystal. Current was applied along an in-plane direction, the magnetic field aligned perpendicular to it within the plane and voltage measured along the $c$ axis.

Here, we want to discuss our results of changes of Hall resistance across the critical pressure. In Fig. 13 we show the Hall resistance $R_{\text {Hall }}$ as a function of pressure for $T=2 \mathrm{~K}$

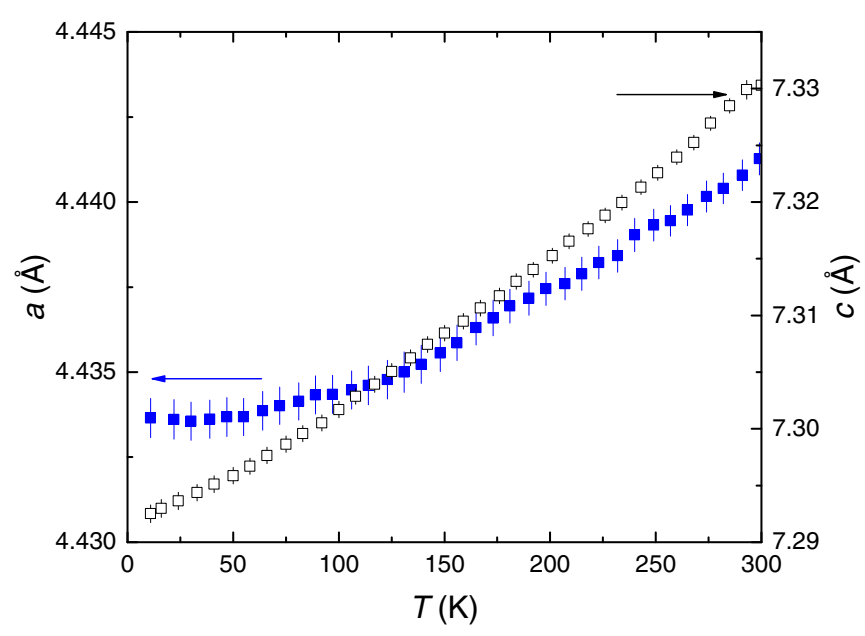

FIG. 14. Lattice parameters $a$ (left axis) and $c$ (right axis) of $\mathrm{EuCd}_{2} \mathrm{As}_{2}$ as a function of temperature $T$ at ambient pressure, determined from $\mathrm{x}$-ray diffraction.

(a),(b) and $T=13 \mathrm{~K}$ (c),(d), i.e., below and above the magnetic transition temperatures $T_{\mathrm{N}}(p)$ and $T_{\mathrm{C}}(p)$. At $2 \mathrm{~K}$ and low pressures, $R_{\text {Hall }}$ goes through a minimum followed by a maximum in a very narrow field range up to $0.8 \mathrm{~T}$. The ordinary Hall effect cannot account for this behavior of $R_{\text {Hall }}$ below $\sim 0.8 \mathrm{~T}$ as it is almost linear [see Fig. 13(b) for data up to higher fields]. In addition, this field scale matches with the one observed in the MR measurements above as well as magnetization measurements at ambient pressure [33]. Thus, this indicates that the low-field Hall effect data are dominated by the anomalous Hall effect contribution. At high pressures, in the FM state, the pronounced maxima and minima are absent and the anomalous contribution to $R_{\text {Hall }}$ is distinctly smaller but nonetheless finite. A full disentanglement of the various contributions to the Hall resistance in a magnetic material is unfortunately not possible on the basis of the present data set given the lack of magnetization data under pressure.

For sufficiently large fields, $\mu_{0} H \gtrsim 4 \mathrm{~T}$, the $R_{\text {Hall }}$ data at $2 \mathrm{~K}$ and $13 \mathrm{~K}$ are linear in field [see Figs. 13(b) and 13(d)] and thus reflects the ordinary Hall contribution. The slope $R_{H}$ obtained from linear fits of the Hall data up to maximum field is plotted as a function for pressure in the insets of Figs. 13(b) and $13(\mathrm{~d})$. For each temperature ( $2 \mathrm{~K}$ and $13 \mathrm{~K}$ ), $R_{H}$ is constant up to $\approx 2 \mathrm{GPa}$ and starts to decrease slightly above. Even though the number of data points at very high pressures, where the decrease becomes pronounced, is limited, our data might indicate a change of charge carrier density across the critical pressure. It will be interesting to investigate the microscopic origin of the change of the magnetic properties with pressure in the future from experimental and theoretical point of view in more detail.

\section{Low-temperature lattice parameters of $\operatorname{EuCd}_{2} \mathrm{As}_{2}$ at ambient pressure}

So as to better compare experimental and theoretical lattice parameters, we determined the lattice parameters of $\mathrm{EuCd}_{2} \mathrm{As}_{2}$ experimentally at low temperatures, given that our 


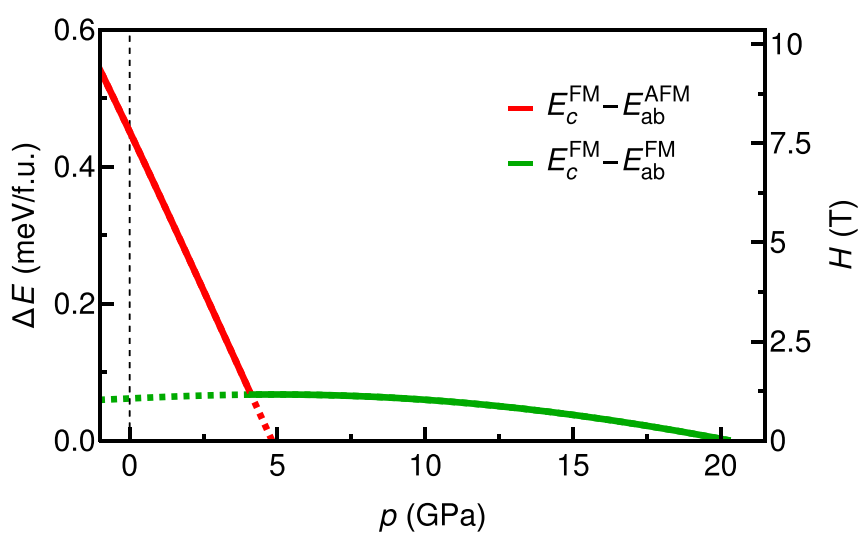

FIG. 15. Energy difference of the ground state compared to $\mathrm{FM}_{c}$ order (left axis) and corresponding magnetic field (right axis) vs pressure. The red curve describes the difference of $\mathrm{AFM}_{a b}$ to $\mathrm{FM}_{c}$, whereas the green curve describes the difference of $\mathrm{FM}_{a b}$ to $\mathrm{FM}_{c}$. Compared to the relaxation types in Fig. 7 and Fig. 8, $p_{c}$ is slightly increased to $4.2 \mathrm{GPa}$ and $p_{c}^{\prime}$, describing the transition to $\mathrm{FM}_{c}$ ground state, decreased to $20 \mathrm{GPa}$.

DFT calculations are performed at zero temperature. Single crystal x-ray diffraction measurements were performed on an in-house four-circle diffractometer using $\mathrm{Cu} K_{\alpha 1}$ radiation from a rotating anode $\mathrm{x}$-ray source, using a germanium

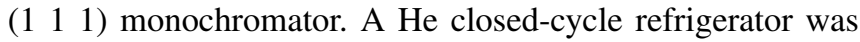
used for temperature dependence measurements between 11 and $300 \mathrm{~K}$. Three Be domes were used as a vacuum shroud, heat shield, and the last innermost dome containing the sample. The innermost dome was filled with a small amount of He gas to improve thermal contact to the sample surface. Measurements were carried out on a single crystal of $0.087 \mathrm{~g}$ attached to a flat copper sample holder that is attached to the cold finger. The mosaicities of the sample were less than $0.04^{\circ}$ for both the $(0,0,5)$ and $(3,0,5)$ reflections at all measured temperatures. The positions of the reflections were fit using a Lorentzian lineshape and used to determine the lattice parameters of the sample from 11-300 K, which are shown in Fig. 14.
As discussed in the main text, the experimental lattice parameters at $11 \mathrm{~K}$ are within $1 \%$ of the calculated values for both the magnetic and the nonmagnetic relaxation. Based on previous works [61], this can be considered as a very good agreement between experiment and theory.

\section{Effect of lattice parameter relaxation in the calculation of energies}

To justify the validity of our calculated data, we used several slightly different calculation settings. Whereas the different calculations resulted in slightly different estimates of the critical pressures, they always resulted in the $\mathrm{AFM}_{a b}$ $\mathrm{FM}_{a b}-\mathrm{FM}_{c}$ transition sequence with increasing pressure. Thus, the key message of our paper is stable with respect to different calculation settings. In the following, we discuss the spread of $p_{c}$ values obtained from different calculation procedures. The order of magnitude for the difference in energy of possible magnetic ground states is already below $1 \mathrm{meV}$ at ambient pressure, for the MAE even below $0.1 \mathrm{meV}$. Therefore, tiny structural changes, caused by different methods of relaxing the unit cell or pseudopotential files, affect the resulting critical pressure $p_{c}$ measurably. Independent of all previously shown calculations, we performed a relaxation using the magnetic space groups $C_{c} 2 / m$, corresponding to the $\mathrm{AFM}_{a b}$, and $C 2 / m, \mathrm{FM}_{a b}$, as starting points for the initial crystal structure. Fixing the Wyckoff positions with regard to the magnetic space groups guarantees no loss of symmetry information. Thereby, we also included SOC and a Hubbard $U=4.4 \mathrm{eV}$. Apart from this, the input parameters are identical to the nonmagnetic case.

Figure 15 illustrates the outcome of this method. Similar to Fig. 8 in the main text, this figure describes the difference of the corresponding ground state energy to the $\mathrm{FM}_{c}$ state as a function of pressure on the left axis and the corresponding magnetic field. The critical pressure $p_{c}$ for the transition from $\mathrm{AFM}_{a b}$ order (red curve) to $\mathrm{FM}_{a b}$ (green curve), given by the crossing point of the red and green curve, is shifted to a slightly larger pressure of $4.2 \mathrm{GPa}$ relative to what is shown in Fig. 8. The predicted transition to $\mathrm{FM}_{c}$ occurs at a lower pressure of $p_{c}^{\prime} \approx 20 \mathrm{GPa}$.
[1] X. Wan, A. M. Turner, A. Vishwanath, and S. Y. Savrasov, Phys. Rev. B 83, 205101 (2011).

[2] M. Z. Hasan and C. L. Kane, Rev. Mod. Phys. 82, 3045 (2010).

[3] B. Yan and C. Felser, Annu. Rev. Condens. Matter Phys. 8, 337 (2017).

[4] A. Burkov, Nat. Mater. 15, 1146 (2016).

[5] S. S.-L. Zhang, A. A. Burkov, I. Martin, and O. G. Heinonen, Phys. Rev. Lett. 123, 187201 (2019).

[6] L. Šmejkal, T. Jungwirth, and J. Sinova, Phys. Status Solidi (RRL) 11, 1700044 (2017).

[7] N. P. Armitage, E. J. Mele, and A. Vishwanath, Rev. Mod. Phys. 90, 015001 (2018).

[8] A. A. Burkov and L. Balents, Phys. Rev. Lett. 107, 127205 (2011).

[9] G. Xu, H. Weng, Z. Wang, X. Dai, and Z. Fang, Phys. Rev. Lett. 107, 186806 (2011).
[10] D. T. Son and B. Z. Spivak, Phys. Rev. B 88, 104412 (2013).

[11] S. A. Parameswaran, T. Grover, D. A. Abanin, D. A. Pesin, and A. Vishwanath, Phys. Rev. X 4, 031035 (2014).

[12] J. Liu and D. Vanderbilt, Phys. Rev. B 90, 155316 (2014).

[13] N. Heinsdorf, M. H. Christensen, M. Iraola, S. Zhang, F. Yang, T. Birol, C. D. Batista, R. Valentí, and R. M. Fernandes, Phys. Rev. B 104, 075101 (2021).

[14] G. Chang, B. Singh, S.-Y. Xu, G. Bian, S.-M. Huang, C.-H. Hsu, I. Belopolski, N. Alidoust, D. S. Sanchez, H. Zheng, H. Lu, X. Zhang, Y. Bian, T. R. Chang, H. T. Jeng, A. Bansil, H. Hsu, S. Jia, T. Neupert, H. Lin, and M. Z. Hasan, Phys. Rev. B 97, 041104(R) (2018).

[15] F. Arnold, C. Shekhar, S.-C. Wu, Y. Sun, R. D. dos Reis, N. Kumar, M. Naumann, M. O. Ajeesh, M. Schmidt, A. G. Grushin et al., Nat. Commun. 7, 11615 (2016). 
[16] H. Weng, C. Fang, Z. Fang, B. A. Bernevig, and X. Dai, Phys. Rev. X 5, 011029 (2015).

[17] S.-M. Huang, S.-Y. Xu, I. Belopolski, C.-C. Lee, G. Chang, B. Wang, N. Alidoust, G. Bian, M. Neupane, C. Zhang et al., Nat. Commun. 6, 7373 (2015).

[18] S. Borisenko, D. Evtushinsky, Q. Gibson, A. Yaresko, K. Koepernik, T. Kim, M. Ali, J. van den Brink, M. Hoesch, A. Fedorov et al., Nat. Commun. 10, 3424 (2019).

[19] E. Liu, Y. Sun, N. Kumar, L. Muechler, A. Sun, L. Jiao, S.-Y. Yang, D. Liu, A. Liang, Q. Xu et al., Nat. Phys. 14, 1125 (2018).

[20] L. Ye, M. Kang, J. Liu, F. von Cube, C. R. Wicker, T. Suzuki, C. Jozwiak, A. Bostwick, E. Rotenberg, D. C. Bell et al., Nature (London) 555, 638 (2018).

[21] M. Kang, L. Ye, S. Fang, J.-S. You, A. Levitan, M. Han, J. I. Facio, C. Jozwiak, A. Bostwick, E. Rotenberg et al., Nat. Mater. 19, 163 (2020).

[22] Z. Guguchia, J. A. T. Verezhak, D. J. Gawryluk, S. S. Tsirkin, J.-X. Yin, I. Belopolski, H. Zhou, G. Simutis, S.-S. Zhang, T. A. Cochran et al., Nat. Commun. 11, 559 (2020).

[23] D. Destraz, L. Das, S. S. Tsirkin, Y. Xu, T. Neupert, J. Chang, A. Schilling, A. G. Grushin, J. Kohlbrecher, L. Keller et al., npj Quantum Mater. 5, 5 (2020).

[24] G. Hua, S. Nie, Z. Song, R. Yu, G. Xu, and K. Yao, Phys. Rev. B 98, 201116(R) (2018).

[25] L.-L. Wang, N. H. Jo, B. Kuthanazhi, Y. Wu, R. J. McQueeney, A. Kaminski, and P. C. Canfield, Phys. Rev. B 99, 245147 (2019).

[26] A. Artmann, A. Mewis, M. Roepke, and G. Michels, Z. Anorg. Allg. Chem. 622, 679 (1996).

[27] H. P. Wang, D. S. Wu, Y. G. Shi, and N. L. Wang, Phys. Rev. B 94, 045112 (2016).

[28] M. C. Rahn, J.-R. Soh, S. Francoual, L. S. I. Veiga, J. Strempfer, J. Mardegan, D. Y. Yan, Y. F. Guo, Y. G. Shi, and A. T. Boothroyd, Phys. Rev. B 97, 214422 (2018).

[29] J.-R. Soh, F. de Juan, M. G. Vergniory, N. B. M. Schröter, M. C. Rahn, D. Y. Yan, J. Jiang, M. Bristow, P. Reiss, J. N. Blandy, Y. F. Guo, Y. G. Shi, T. K. Kim, A. McCollam, S. H. Simon, Y. Chen, A. I. Coldea, and A. T. Boothroyd, Phys. Rev. B 100, 201102(R) (2019).

[30] J.-Z. Ma, S. M. Nie, C. J. Yi, J. Jandke, T. Shang, M. Y. Yao, M. Naamneh, L. Q. Yan, Y. Sun, A. Chikina et al., Sci. Adv. 5, eaaw4718 (2019).

[31] C. Niu, N. Mao, X. Hu, B. Huang, and Y. Dai, Phys. Rev. B 99, 235119 (2019).

[32] Y. Xu, L. Das, J. Z. Ma, C. J. Yi, S. M. Nie, Y. G. Shi, A. Tiwari, S. S. Tsirkin, T. Neupert, M. Medarde, M. Shi, J. Chang, and T. Shang, Phys. Rev. Lett. 126, 076602 (2021).

[33] N. H. Jo, B. Kuthanazhi, Y. Wu, E. Timmons, T.-H. Kim, L. Zhou, L.-L. Wang, B. G. Ueland, A. Palasyuk, D. H. Ryan, R. J. McQueeney, K. Lee, B. Schrunk, A. A. Burkov, R. Prozorov, S. L. Budko, A. Kaminski, and P. C. Canfield, Phys. Rev. B 101, 140402(R) (2020).
[34] L. D. Sanjeewa, J. Xing, K. M. Taddei, D. Parker, R. Custelcean, C. dela Cruz, and A. S. Sefat, Phys. Rev. B 102, 104404 (2020).

[35] K. Taddei, L. Lin, L. Sanjeewa, J. Xing, C. dela Cruz, A. Sefat, and D. Parker, arXiv:2012.01555.

[36] N. Jo, Y. Wu, T. Trevisan, L.-L. Wang, K. Lee, B. Kuthanazhi, B. Schrunk, S. L. Bud'ko, P. C. Canfield, P. P. Orth et al., arXiv:2109.08538.

[37] P. C. Canfield, T. Kong, U. S. Kaluarachchi, and N. H. Jo, Philos. Mag. 96, 84 (2016).

[38] https://lspceramics.com/canfield-crucible-sets-2/.

[39] P. C. Canfield, Rep. Prog. Phys. 83, 016501 (2019).

[40] E. Gati, G. Drachuck, L. Xiang, L.-L. Wang, S. L. Bud'ko, and P. C. Canfield, Rev. Sci. Instrum. 90, 023911 (2019).

[41] S. L. Bud'ko, A. N. Voronovskii, A. G. Gapotchenko, and E. S. ltskevich, Zh. Eksp. Teor. Fiz. 86, 778 (1984).

[42] M. S. Torikachvili, S. K. Kim, E. Colombier, S. L. Bud'ko, and P. C. Canfield, Rev. Sci. Instrum. 86, 123904 (2015).

[43] A. Eiling and J. S. Schilling, J. Phys. F: Met. Phys. 11, 623 (1981).

[44] R. Khasanov, Z. Guguchia, A. Maisuradze, D. Andreica, M. Elender, A. Raselli, Z. Shermadini, T. Goko, F. Knecht, E. Morenzoni et al., High Press. Res. 36, 140 (2016).

[45] T. F. Smith and C. W. Chu, Phys. Rev. 159, 353 (1967).

[46] P. Hohenberg and W. Kohn, Phys. Rev. 136, B864 (1964).

[47] W. Kohn and L. J. Sham, Phys. Rev. 140, A1133 (1965).

[48] J. P. Perdew, K. Burke, and M. Ernzerhof, Phys. Rev. Lett. 77, 3865 (1996).

[49] P. E. Blöchl, Phys. Rev. B 50, 17953 (1994).

[50] G. Kresse and D. Joubert, Phys. Rev. B 59, 1758 (1999).

[51] G. Kresse and J. Hafner, Phys. Rev. B 47, 558 (1993).

[52] G. Kresse and J. Furthmüller, Phys. Rev. B 54, 11169 (1996).

[53] G. Kresse and J. Furthmüller, Comput. Mater. Sci. 6, 15 (1996).

[54] H. J. Monkhorst and J. D. Pack, Phys. Rev. B 13, 5188 (1976).

[55] M. E. Fisher and J. S. Langer, Phys. Rev. Lett. 20, 665 (1968).

[56] V. Taufour, U. S. Kaluarachchi, R. Khasanov, M. C. Nguyen, Z. Guguchia, P. K. Biswas, P. Bonfà, R. De Renzi, X. Lin, S. K. Kim, E. D. Mun, H. Kim, Y. Furukawa, C. Z. Wang, K. M. Ho, S. L. Budko, and P. C. Canfield, Phys. Rev. Lett. 117, 037207 (2016).

[57] E. Gati, J. M. Wilde, R. Khasanov, L. Xiang, S. Dissanayake, R. Gupta, M. Matsuda, F. Ye, B. Haberl, U. Kaluarachchi, R. J. McQueeney, A. Kreyssig, S. L. Budko, and P. C. Canfield, Phys. Rev. B 103, 075111 (2021).

[58] R. Khasanov, A. Amato, P. Bonfà, Z. Guguchia, H. Luetkens, E. Morenzoni, R. D. Renzi, and N. D. Zhigadlo, J. Phys.: Condens. Matter 29, 164003 (2017).

[59] F. Birch, Phys. Rev. 71, 809 (1947).

[60] F. D. Murnaghan, Proc. Natl. Acad. Sci. USA 30, 244 (1944).

[61] J. Harl, L. Schimka, and G. Kresse, Phys. Rev. B 81, 115126 (2010).

[62] Z. Shermadini, R. Khasanov, M. Elender, G. Simutis, Z. Guguchia, K. Kamenev, and A. Amato, High Press. Res. 37, 449 (2017). 